\title{
Renewal-type Limit Theorem for Continued Fractions with Even Partial Quotients
}

\author{
Francesco Cellarosi*
}

August 5, 2008

\begin{abstract}
We prove the existence of the limiting distribution for the sequence of denominators generated by continued fraction expansions with even partial quotients, which were introduced by $\mathrm{F}$. Schweiger [14] [15] and studied also by C. Kraaikamp and A. Lopes [11. Our main result is proven following the strategy used by Ya. Sinai and C. Ulcigrai 18 in their proof of a similar renewal-type theorem for Euclidean continued fraction expansions and the Gauss map. The main steps in our proof are the construction of a natural extension of a Gauss-like map and the proof of mixing of a related special flow.
\end{abstract}

\section{Introduction}

Continued fractions with even partial quotients (ECF) were originally introduced by F. Schweiger [14] [15] and appeared to be qualitatively different from the usual Euclidean continued fractions. In order to study ECF-expansions from a dynamical point of view, one should define a Gauss-like map $T$ and its associated jump transformation $R$, which is used to overcome the intermittent behavior of $T$. Let $\left\{q_{n}\right\}_{n \in \mathbb{N}}$ be the sequence of the denominators of the ECF-convergents. For $L>0$ define the renewal time $n_{L}=\min \left\{n \in \mathbb{N}: q_{n}>L\right\}$. Our main result is a renewal-type limit theorem which establishes the existence of a limiting distribution for $\frac{q_{n_{L}}}{L}$, jointly with any finite number of entries preceeding and following the renewal time $n_{L}$ when $L \rightarrow \infty$.

With the help of the map $R$, we construct a subsequence $\left\{\hat{q}_{n}\right\}_{n \in \mathbb{N}} \subset\left\{q_{n}\right\}_{n \in \mathbb{N}}$ and define the renewal time $\hat{n}_{L}=\min \left\{n \in \mathbb{N}: \hat{q}_{n}>L\right\}$. The main result follows from another renewal-type theorem, which shows the existence of a limiting distribution for $\frac{\hat{q}_{\hat{n}_{L}}}{L}$. The proof of this theorem exploits the mixing properties of a suitably defined special flow over the natural extension of $R$, following the strategy used by Ya. Sinai and C. Ulcigrai [18].

The paper is organized as follows: in Section 1 we introduce ECF-expansions comparing them with Euclidean continued fraction expansions. After introducing the maps $T$ and $R$ and the sequences of denominators $\left\{\hat{q}_{n}\right\}_{n \in \mathbb{N}} \subset\left\{q_{n}\right\}_{n \in \mathbb{N}}$, we formulate our two renewal-type theorems (concerning $\left\{q_{n}\right\}_{n \in \mathbb{N}}$ and $\left\{\hat{q}_{n}\right\}$ respectively) and present the main tools (natural extensions and special

\footnotetext{
*Mathematics Department, Princeton University. fcellaro@math.princeton.edu
} 
flows). Section 2 is devoted to estimating the growth of the sequence $\left\{\hat{q}_{n}\right\}_{n \in \mathbb{N}}$. In Section 3 we define a special flow over the natural extension of $R$ and show how it is used to approximate the logarithm of the $R$-denominators $\hat{q}_{n}$. The mixing property of the flow is shown in Appendix A. Further lemmata are proven in Section 3, allowing us to "localize" the problem to sufficiently small cylinders. Section 4 contains the proof of the renewal-type theorem for $\left\{\hat{q}_{n}\right\}_{n \in \mathbb{N}}$, which requires all previous lemmata and, as its corollary, we prove our Main Theorem. As the anonymous referee pointed out, the technique of the present paper could be also suitably adapted for proving the renewal theorem for denominators of the best approximations of the first kind which are associated with the Farey map.

\section{Preliminaries and Main Result}

\subsection{Euclidean Continued Fractions and the Gauss map}

Given $\alpha \in(0,1] \backslash \mathbb{Q}$, its continued fraction expansion is denoted by

$$
\alpha=\frac{1}{a_{1}+\frac{1}{a_{2}+\frac{1}{a_{3}+\frac{1}{\ddots}}}}=\left[a_{1}, a_{2}, a_{3}, \ldots, a_{n}, \ldots\right],
$$

where $a_{n} \in \mathbb{N}=\{1,2,3, \ldots\}$. The convergents of $\alpha$ are denoted by $\left\{P_{n} / Q_{n}\right\}_{n \in \mathbb{N}}$ and defined by $\frac{P_{n}}{Q_{n}}=\left[a_{1}, a_{2}, \ldots, a_{n}\right]$, with $\operatorname{GCD}\left(P_{n}, Q_{n}\right)=1$. This kind of continued fractions is called "Euclidean" because of its connection with the Euclidean algorithm used to determine the GCD of two positive integers: given $a, b \in \mathbb{N}, a<b$, the partial quotients given by the Euclidean algorithm are the entries of the continued fraction expansion of $\alpha=\frac{a}{b}$.

Let $G$ be the Gauss map, i.e. $G:(0,1] \rightarrow(0,1], G(\alpha)=\left\{\frac{1}{\alpha}\right\}=\frac{1}{\alpha}-\left\lfloor\frac{1}{a}\right\rfloor$, where $\{\cdot\}$ and $\lfloor\cdot\rfloor$ denote the fractional and the integer part respectively. The sequence $\left\{a_{n}\right\}_{n \in \mathbb{N}}$ represents a symbolic coding of the orbit $\left\{G^{n}(\alpha)\right\}_{n \in \mathbb{N}}$, namely $a_{n}=\left\lfloor\frac{1}{G^{n-1}(\alpha)}\right\rfloor$. A point $\alpha \in(0,1] \backslash \mathbb{Q}$ is thus identified with the sequence $\left\{a_{n}\right\}_{n \in \mathbb{N}} \in \mathbb{N}^{\mathbb{N}}$. There is a natural Markov partition of $(0,1]$ for the Gauss map corresponding to this symbolic representation. $G$ has countably many branches; each branch is surjective and defined on $A(k)=\left(\frac{1}{k+1}, \frac{1}{k}\right]$, by $\left.G\right|_{A(k)}(\alpha)=\frac{1}{\alpha}-k$. The map $G$ has an invariant measure $\mu_{G}$ which is absolutely continuous w.r.t. the Lebesgue measure on $(0,1]$ and it is given by the density $\frac{\mathrm{d} \mu_{G}}{\mathrm{~d} \alpha}=\frac{1}{\log 2} \frac{1}{1+\alpha}$. As a general reference, see [10].

\subsection{Continued Fractions with Even Entries and the corresponding map $T$}

We shall consider a modification of the Euclidean continued fraction expansion of $\alpha \in(0,1] \backslash \mathbb{Q}$, namely an expression of the form

$$
\alpha=\frac{1}{2 k_{1}+\frac{\xi_{1}}{2 k_{2}+\frac{\xi_{2}}{2 k_{3}+\frac{\xi_{3}}{\ddots}}}}=\left[\left[\left(k_{1}, \xi_{1}\right),\left(k_{2}, \xi_{2}\right),\left(k_{3}, \xi_{3}\right), \ldots,\left(k_{n}, \xi_{n}\right), \ldots\right]\right],
$$


where $\left(k_{n}, \xi_{n}\right) \in \mathbb{N} \times\{ \pm 1\}=: \Omega$. The corresponding convergents of $\alpha$ are denoted by $\left\{p_{n} / q_{n}\right\}_{n \in \mathbb{N}}$ and defined by

$$
\frac{p_{n}}{q_{n}}=\left[\left[\left(k_{1}, \xi_{1}\right),\left(k_{2}, \xi_{2}\right), \ldots,\left(k_{n}, *\right)\right]\right]=\frac{1}{2 k_{1}+\frac{\xi_{1}}{2 k_{2}+\frac{\xi_{2}}{2 k_{3}+\ldots+\frac{\xi_{n-2}}{2 k_{n-1}+\frac{\xi_{n-1}}{2 k_{n}}}}}},
$$

where $\operatorname{GCD}\left(p_{n}, q_{n}\right)=1$ and "*" denotes an arbitrary element of $\{ \pm 1\}$. The algorithm which corresponds to this kind of continued fractions is similar to the Euclidean one, but at each step it is of the form $v=c u+\xi r$, with $c \in 2 \mathbb{N}, \xi= \pm 1$ and $0 \leq r<u$. Given $a, b \in \mathbb{N}, a<b$, as input, the sequence of pairs $\left\{\left(c_{n}, \xi_{n}\right)\right\}_{n \in \mathbb{N}}$ generated by the algorithm corresponds to the sequence $\left\{\left(k_{n}, \xi_{n}\right)\right\}_{n \in \mathbb{N}}$ relative to expansion of $\alpha=\frac{a}{b}$, with $k_{n}=\frac{c_{n}}{2}$. In this context, the role of the Gauss map is played by the map $T$, defined as follows. Let us consider the partition of the interval $(0,1]$ given by $\{B(k, \xi)\}_{(k, \xi) \in \Omega}$, where

$$
B(k,-1)=\left(\frac{1}{2 k}, \frac{1}{2 k-1}\right], \quad B(k,+1)=\left(\frac{1}{2 k+1}, \frac{1}{2 k}\right], \quad k \in \mathbb{N}
$$

and let $T$ be the map on $(0,1]$ given by

$$
T(x)=\xi \cdot\left(\frac{1}{x}-2 k\right), \quad x \in B(k, \xi) .
$$

Notice that for $x \in B(k, \xi)$ we have the identity $x=\frac{1}{2 k+\xi T(x)}$. Therefore

$$
x=\left[\left[\left(k_{1}, \xi_{1}\right),\left(k_{2}, \xi_{2}\right),\left(k_{3}, \xi_{3}\right), \ldots\right]\right] \quad \text { implies } T^{n}(x)=\left[\left[\left(k_{n+1}, \xi_{n+1}\right),\left(k_{n+2}, \xi_{n+2}\right), \ldots\right]\right],
$$

i.e $T$ acts as a shift over the space $\Omega^{\mathbb{N}}$. Continued fraction expansions with even partial quotients and their connection with the map $T$ were initially discussed by F. Schweiger [14, 15] and a deep insight was provided by C. Kraaikamp and A. Lopes [11], in relation with closed geodesics for the theta group (the subgroup of $\mathrm{SL}(2, \mathbb{Z})$ generated by $z \mapsto-\frac{1}{z}$ and $z \mapsto z+2$ ). A detailed analysis of the Euclidean-like algorithm associated to ECF-expansions (and several other expansions) is presented by B. Vallée [19].

Our interest for such continued fraction expansions and the map $T$ comes from the study of quadratic trigonometric sums of the form $\sum_{n=0}^{N-1} \exp \left(\pi i \alpha n^{2}\right)$, whose renormalization properties are described (see e.g. [3, 6, 8]) by the transformation on $[-1,1] \backslash\{0\}$ defined by $x \mapsto-\frac{1}{x}(\bmod 2)$. The restriction of this map to $(0,1]$ is, in modulus, equal to the map $T$. We shall not dwell on the renormalization of such sums, which will be the subject of our future work.

In comparison with the Gauss map $G$, the map $T$ has a different qualitative behavior. Indeed, it is not uniformly expanding since it has an indifferent fixed point 1 at $x=1$. Because of this, the map $\mathrm{T}$ does not have a finite invariant measure absolutely continuous w.r.t. the Lebesgue measure.

\footnotetext{
${ }^{1} \mathrm{~A}$ fixed point $x_{0}$ is called indifferent if the map has derivative equal to 1 in modulus at $x_{0}$. This feature can be found in several maps, which are called intermittent. A celebrated example is the Farey map 9], which has an indifferent fixed point at 0 . For an overview on intermittent systems see, e.g., 13].
} 


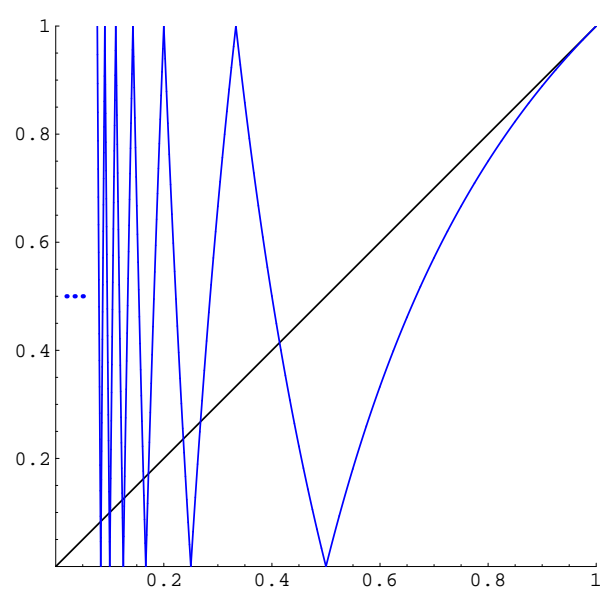

Figure 1: Graph of $T$.

Indeed the expectation of the time spent by a random trajectory in a small neighborhood of $x=1$ is infinite and therefore any invariant measure should give infinite measure to any neighborhood of $x=1$. The following Theorem was proven by F. Schweiger [14]:

Theorem 1.1. The map $T:(0,1] \rightarrow(0,1]$ has a $\sigma$-finite invariant measure $\nu$ with infinite mass. $\nu$ has density $h(\alpha)=\frac{1}{\alpha+1}-\frac{1}{\alpha-1}$. Moreover $T$ is ergodic, i.e. if $A$ is a $T$-invariant set, then either $\nu(A)=0$ or $\nu\left(A^{c}\right)=0$.

Remark 1.2. It is easy to convert Euclidean continued fraction expansions into ECF-expansions, using the following identity:

$$
a_{1}+\frac{1}{a_{2}+\frac{1}{a_{3}+\gamma}}=\left(a_{1}+1\right)-\frac{1}{2-\frac{1}{2-\ldots-\frac{1}{2-\frac{1}{\left(a_{3}+1\right)+\gamma}}}},
$$

where "2" appears $a_{2}-1$ times in the right hand side. Given $\alpha=\left[a_{1}, a_{2}, a_{3}, \ldots\right]$, we can recursively apply the previous identity, moving from the left to the right, to each triplet $\left(a_{j-1}, a_{j}, a_{j+1}\right)$ such that $a_{j-1}$ is odd and obtain a new sequence $\left\{\left(c_{n}, \xi_{n}\right)\right\}_{n \in \mathbb{N}}$ with $c_{n} \in 2 \mathbb{N}$ and $\xi_{n} \in\{ \pm 1\}$. Setting $k_{n}=\frac{c_{n}}{2}$, we get the sequence $\left\{\left(k_{n}, \xi_{n}\right)\right\}_{n \in \mathbb{N}}$ such that $\alpha=\left[\left[\left(k_{1}, \xi_{1}\right),\left(k_{2}, \xi_{2}\right),\left(k_{3}, \xi_{3}\right), \ldots\right]\right]$. It is easy to see that $1=[[(1,-1),(1,-1), \ldots]]$ and in particular (1) shows that the ECF-expansion of any rational number is either finite or eventually periodic with $\overline{(1,-1)}$-tail. In our discussion we shall deal only with irrational $\alpha$ for which the ECF-expansion is infinite with no $\overline{(1,-1)}$-tail. Let us denote the set of such sequences with $\dot{\Omega}^{\mathbb{N}}$.

\subsection{The Jump Transformation $R$}

Let $\alpha \in(0,1] \backslash \mathbb{Q}=\dot{\Omega}^{\mathbb{N}}$, which is endowed with the Borel $\sigma$-algebra $\mathfrak{B}$. We use the notion of jump transformation, due to Schweiger (see [16], chapter 19), to construct an uniformly expanding 
$\operatorname{map} R:(0,1] \backslash \mathbb{Q} \longrightarrow(0,1] \backslash \mathbb{Q}$. Define

$$
\begin{aligned}
\tau(\alpha) & :=\min \left\{j \geq 0 \text { s.t. } T^{j}(\alpha) \in B(1,-1)^{c}=\left(0, \frac{1}{2}\right]\right\} \text { and } \\
R(\alpha) & :=T^{\tau(\alpha)+1}(\alpha) .
\end{aligned}
$$

$R$ is said to be the jump transformation 2 associated to $T$ w.r.t. $\left(0, \frac{1}{2}\right]$. It can be checked that

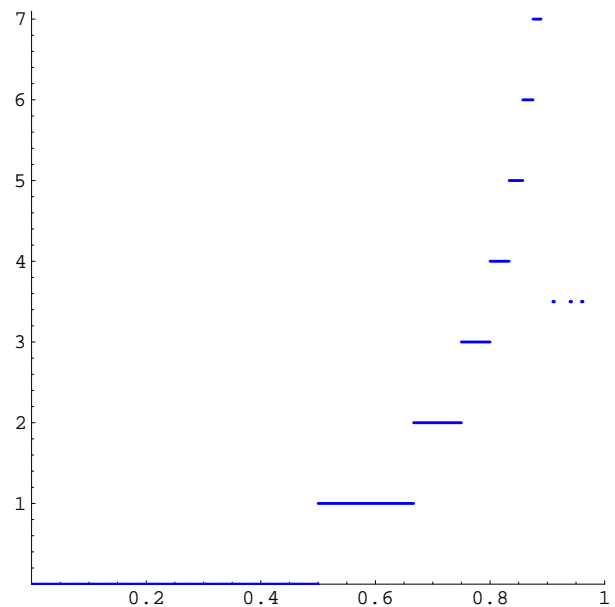

Figure 2: Graph of $\tau$.

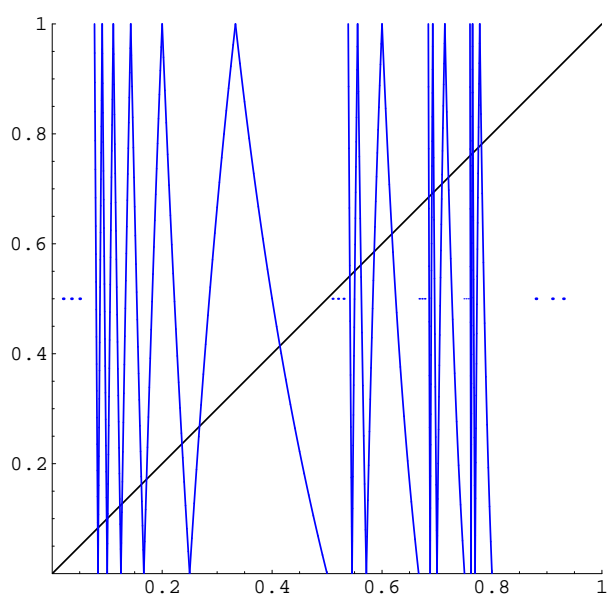

Figure 3: Graph of $R$.

$R$ is uniformly expanding and has bounded distortion, more precisely $\inf _{\alpha \in(0,1] \backslash \mathbb{Q}}\left|R^{\prime}(\alpha)\right| \geq 4$ and $\sup _{\alpha \in(0,1] \backslash \mathbb{Q}}\left|R^{\prime \prime}(\alpha) /\left(R^{\prime}(\alpha)\right)^{2}\right| \leq 2$. Therefore, since its branches are surjective, we can deduce by the "Folklore Theorem" of Adler [1, 4] that it has an invariant probability measure $\mu$ which is absolutely continuous w.r.t. the Lebesgue measure. Its density $f=\frac{\mathrm{d} \mu}{\mathrm{d} \alpha}$, can be computed explicitly (e.g. using transfer operator identities, [19]):

$$
f(\alpha)=\frac{1}{\log 3}\left(\frac{1}{3-\alpha}+\frac{1}{1+\alpha}\right) .
$$

Let us construct the symbolic representation of the map $R$. Denote $\bar{\omega}=(1,-1) \in \Omega$ and $\Omega^{*}:=$ $\Omega \backslash\{\bar{\omega}\}$. Given $\alpha=\left[\left[\omega_{1}, \omega_{2}, \omega_{3}, \ldots\right]\right] \in \dot{\Omega}^{\mathbb{N}}$ we have that $\tau=\tau(\alpha)=\min \left\{j \geq 0\right.$ s.t. $\left.\omega_{j+1} \neq \bar{\omega}\right\}$ and $R(\alpha)=\left[\left[\omega_{\tau+2}, \omega_{\tau+3}, \omega_{\tau+4}, \ldots\right]\right]$. Equivalently,

$$
\begin{gathered}
{\left[\left[\omega_{1}, \omega_{2}, \omega_{3}, \ldots\right]\right] \stackrel{R}{\longmapsto}\left[\left[\omega_{2}, \omega_{3}, \omega_{4}, \ldots\right]\right] \quad \text { if } \omega_{1} \in \Omega^{*}} \\
{\left[[\underbrace{\bar{\omega}, \ldots, \bar{\omega}}_{k \text { times }}, \omega_{k+1}, \omega_{k+2}, \omega_{k+3}, \ldots]\right] \stackrel{R}{\longmapsto}\left[\left[\omega_{k+2}, \omega_{k+3}, \ldots\right]\right] \quad \text { if } \omega_{k+1} \in \Omega^{*} .}
\end{gathered}
$$

Let us set $\Sigma=\mathbb{N}_{0} \times \Omega^{*}, \mathbb{N}_{0}=\mathbb{N} \cup\{0\}$, and denote by $(h, \omega) \in \Sigma$ the word $(\underbrace{\bar{\omega}, \ldots \bar{\omega}}_{h \text { times }}, \omega)$ of length $h+1$ where $\omega \in \Omega^{*}$. In this way we code each element of $\dot{\Omega}^{\mathbb{N}}$ by an element of $\Sigma^{\mathbb{N}}$ and this coding is

\footnotetext{
${ }^{2}$ Some authors refer to this map as the induced map (or the first passage map) w.r.t (0, $\left.\frac{1}{2}\right]$.
} 
clearly invertible. After we identify $(0,1] \backslash \mathbb{Q}$ with $\Sigma^{\mathbb{N}}$, the map $R:(0,1] \backslash \mathbb{Q} \rightarrow(0,1] \backslash \mathbb{Q}$ becomes a shift on the space $\Sigma^{\mathbb{N}}$.

For brevity, we denote $m^{ \pm}=0 \cdot m^{ \pm}=(0,(m, \pm 1))$ and $h \cdot m^{ \pm}=(h,(m, \pm 1))$. By construction, $h \cdot 1^{-}$is not allowed for any $h \in \mathbb{N}_{0}$. In the following, we will use both the codings $(0,1] \backslash \mathbb{Q}=\dot{\Omega}^{\mathbb{N}}$ and $(0,1] \backslash \mathbb{Q}=\Sigma^{\mathbb{N}}$, denoting the elements of $\Omega$ by $\omega$ and the ones of $\Sigma$ by $\sigma$.

Example 1.3.

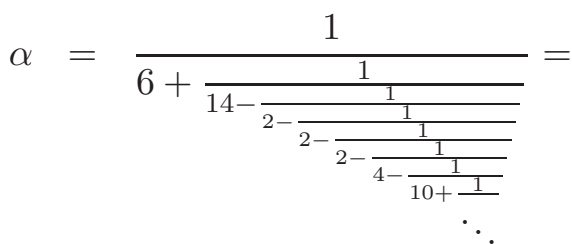

$$
\begin{aligned}
& =((3,+1),(7,-1),(1,-1),(1,-1),(1,-1),(2,-1),(5,+1), \ldots) \in \dot{\Omega}^{\mathbb{N}} \\
& =((0,(3,+1)),(0,(7,-1)),(3,(2,-1)),(0,(5,+1)), \ldots) \in \Sigma^{\mathbb{N}} \\
& =\left(0 \cdot 3^{+}, 0 \cdot 7^{-}, 3 \cdot 2^{-}, 0 \cdot 5^{+}, \ldots\right)=\left(3^{+}, 7^{-}, 3 \cdot 2^{-}, 5^{+}, \ldots\right) \text {. }
\end{aligned}
$$

\subsection{Natural Extension of $R$}

The notion of natural extension of was introduced by V. Rokhlin [12] and since then it became a powerful tool in the study of metric invariants and statistical properties of endomorphisms of measure spaces. Since $R$ is a shift over $\Sigma^{\mathbb{N}}$, it is easy to construct its natural extension $\hat{R}$, which will act on the space $D(\hat{R}):=\Sigma^{\mathbb{Z}}$ :

$$
\left(\ldots, \sigma_{-2}, \sigma_{-1}, \sigma_{0} ; \sigma_{1}, \sigma_{2}, \ldots\right) \stackrel{\hat{R}}{\longmapsto}\left(\ldots, \sigma_{-2}^{\prime}, \sigma_{-1}^{\prime}, \sigma_{0}^{\prime} ; \sigma_{1}^{\prime}, \sigma_{2}^{\prime}, \ldots\right)
$$

where $\sigma_{i}^{\prime}=\sigma_{i+1}$. The map $\hat{R}$ is clearly invertible and we define the $\sigma$-algebra $\hat{\mathfrak{B}}$ on $D(\hat{R})$ as the smallest $\sigma$-algebra containing the preimages $T^{-1}(C), C \in \mathfrak{B}$.

Given any $\hat{\omega} \in D(\hat{R}), \hat{\omega}^{-}=\left(\sigma_{0}, \sigma_{-1}, \sigma_{-2}, \ldots\right)$ and $\hat{\omega}^{+}=\left(\sigma_{1}, \sigma_{2}, \sigma_{3}, \ldots\right)$ will denote the two components of $\hat{\omega}$, with $\sigma_{i} \in \Sigma, i \in \mathbb{Z}$. Moreover, $\hat{R}$ has an invariant measure $\hat{\mu}$ which is obtained by setting

$$
\hat{\mu}\left(\left\{\hat{\omega} \in D(\hat{R}): \sigma_{i_{1}} \in C_{1}, \ldots, \sigma_{i_{r}} \in C_{r}\right\}\right):=\mu\left(\left\{\hat{\omega}^{+} \in \Sigma^{\mathbb{N}}: \sigma_{i_{1}+n} \in C_{1}, \ldots, \sigma_{i_{r}+n} \in C_{r}\right\}\right),
$$

for any $r \in \mathbb{N}, i_{1}, \ldots, i_{r} \in \mathbb{Z}$ and $C_{1}, \ldots, C_{r} \in \mathfrak{B}$, where $n \geq 0$ is chosen such that $i_{k}+n>0$ for $k=1, \ldots, r$.

Remark 1.4. Coding $\left(\ldots, \sigma_{-1}, \sigma_{0} ; \sigma_{1}, \sigma_{2}, \ldots\right)$ into $\left(\ldots, \omega_{-1}, \omega_{0} ; \omega_{1}, \omega_{2}, \ldots\right)$ we get $\alpha=\hat{\omega}^{+}=$ $\left[\left[\omega_{1}, \omega_{2}, \omega_{3} \ldots\right]\right] \in(0,1] \backslash \mathbb{Q}$. On the other hand, we can identify $\hat{\omega}^{-}$with a point in $\left(-\frac{1}{3}, 1\right] \backslash \mathbb{Q}$ by setting

$$
\hat{\omega}^{-}=\left(\omega_{0}, \omega_{-1}, \omega_{-2}, \ldots\right)=\left[\left[\left(0, \xi_{0}\right) ;\left(k_{0}, \xi_{-1}\right),\left(k_{-1}, \xi_{-2}\right), \ldots\right]\right]=\frac{\xi_{0}}{2 k_{0}+\frac{\xi_{-1}}{2 k_{-1}+\frac{\xi_{-2}}{\ddots}}} .
$$

Therefore $D(\hat{R})$ is identified with the "rectangle" $(0,1] \times\left(-\frac{1}{3}, 1\right] \backslash \mathbb{Q}^{2}$. 
The natural extension of the map $T$, denoted by $\hat{T}$, is defined analogously as an invertible shift over $\Omega^{\mathbb{Z}}$, the space of bi-sided sequences over the alphabet $\Omega$. Schweiger [15] proved that $\hat{T}$ has an absolutely continuous invariant measure over $(0,1] \times(-1,1] \backslash \mathbb{Q}^{2}$ which is $\sigma$-finite with infinite mass.

\section{$1.5 R$-Convergents}

For $\hat{\omega} \in D(\hat{R})$ we set $p_{n}(\hat{\omega})=p_{n}\left(\hat{\omega}^{+}\right), q_{n}(\hat{\omega})=q_{n}\left(\hat{\omega}^{+}\right)$and we call $\frac{p_{n}}{q_{n}}$ the $n$-th $T$-convergent of $\hat{\omega}$. We are interested in a particular subsequence of $\left\{p_{n} / q_{n}\right\}_{n \in \mathbb{N}}$, corresponding to the map $R$, defined as follows.

Set $\theta_{0}=1$ and $\theta_{i}=\theta_{i}(\hat{\omega})=\theta_{i}\left(\hat{\omega}^{+}\right)=1+\tau\left(R^{i-1}\left(\hat{\omega}^{+}\right)\right)$for $i \geq 1$ and define $\nu_{n}=\nu_{n}(\hat{\omega})=$ $\nu_{n}\left(\hat{\omega}^{+}\right)=\sum_{k=0}^{n} \theta_{i}, n \geq 0$. The sequence $\left\{\nu_{n}\right\}_{n}$ gives us the index in $\hat{\omega}$ of the first $\Omega$-coordinate of $\hat{R}^{n}(\hat{\omega})$, i.e. $\left(\hat{R}^{n}\left(\ldots, \omega_{0} ; \omega_{1}, \omega_{2}, \ldots\right)\right)^{+}=\left(\omega_{\nu_{n}}, \omega_{\nu_{n}+1}, \ldots\right), \omega_{i} \in \Omega$. Furthermore, we have the useful recurrent relation:

$$
\nu_{n}(\hat{\omega})=\nu_{n-1}(\hat{\omega})+\tau\left(R^{n-1}\left(\hat{\omega}^{+}\right)\right)+1
$$

The fraction $\frac{p_{\nu_{n}}}{q_{\nu_{n}}}$ is called the $n$-th $R$-convergent of $\hat{\omega}$. We are mainly concerned about the sequence of denominators of the $R$-convergents of $\hat{\omega}$, i.e. $\hat{q}_{n}=\hat{q}_{n}(\hat{\omega})=\hat{q}_{n}\left(\hat{\omega}^{+}\right):=q_{\nu_{n}}, n \in \mathbb{N}$. The 0 -th denominator is defined in a different way, namely $\hat{q}_{0}:=1$. Notice that $\nu_{0}(\hat{\omega})=1$ for each $\hat{\omega} \in D(\hat{R})$ and $1=\hat{q}_{0} \neq q_{\nu_{0}}=2 k_{1} \geq 2$. It is possible to define $\nu_{-n}=\nu_{-n}(\hat{\omega})=\nu_{-n}\left(\hat{\omega}^{-}\right)$for $n \in \mathbb{N}$ as well, namely $\nu_{-n}=-\sum_{k=1}^{n} \tau\left(\left(\hat{R}^{-k}(\hat{\omega})\right)^{+}\right)-n+1$.

Example 1.5. Let $\alpha=\hat{\omega}^{+}=\pi-3$.

$$
\begin{aligned}
\alpha=\hat{\omega}^{+} & =(4^{-}, \underbrace{1^{-}, \ldots, 1^{-}}_{14 \text { times }}, 1^{+}, 146^{+}, 1^{-}, 1^{+}, 1^{+}, 1^{-}, 1^{-}, 1^{-}, 1^{+}, 7^{+}, 2^{+}, \ldots)= \\
& =\left(4^{-}, 14 \cdot 1^{+}, 146^{+}, 1 \cdot 1^{+}, 1^{+}, 3 \cdot 1^{+}, 7^{+}, 2^{+}, \ldots\right),
\end{aligned}
$$

We have $\left\{\theta_{k}\right\}_{k=0}^{8}=\{1,1,15,1,2,1,4,1,1\}$ and hence $\left\{\nu_{n}\right\}_{n=0}^{8}=\{1,2,17,18,20,21,25,26,27\}$.

\subsection{Main Result: Renewal-type Theorems for $\left\{q_{n}\right\}$ and $\left\{\hat{q}_{n}\right\}$}

Given $L>0$, consider the smallest index $n$ for which $q_{n}$ exceeds $L$, namely

$$
n_{L}=n_{L}(\hat{\omega})=n_{L}\left(\hat{\omega}^{+}\right):=\min \left\{n \in \mathbb{N}: q_{n}>L\right\},
$$

which is referred to as renewal time or waiting time. The following Theorem is the main result of this paper.

Main Theorem. Fix $N_{1}, N_{2} \in \mathbb{N}$. The ratio $\frac{q_{n_{L}}}{L}$ and the entries $\omega_{n_{L}+j}$ for $-N_{1}<j \leq N_{2}$ have a joint limiting probability distribution, as $L \rightarrow \infty$, with respect to the measure $\mu$. In other words: for each $N_{1}, N_{2} \in \mathbb{N}$ there exists a probability measure $\mathrm{P}_{N_{1}, N_{2}}$ on $(1,+\infty) \times \Omega^{N_{1}+N_{2}}$ such that for all $a, b>1, d_{j} \in \Omega,-N_{1}<j \leq N_{2}$,

$$
\begin{array}{r}
\mu\left(\left\{\alpha: a<\frac{q_{n_{L}}}{L}<b, \omega_{n_{L}+j}=d_{j},-N_{1}<j \leq N_{2}\right\}\right) \stackrel{L \rightarrow \infty}{\longrightarrow} \\
\mathrm{P}_{N_{1}, N_{2}}\left((a, b) \times\left\{d_{-N_{1}+1}\right\} \times \cdots \times\left\{d_{0}\right\} \times \cdots \times\left\{d_{N_{2}}\right\}\right) .
\end{array}
$$


A similar statement was proven by Ya. Sinai and C. Ulcigrai [18], for the Gauss map and the denominators $\left\{Q_{n}\right\}_{n \in \mathbb{N}}$. They used a special flow and its mixing property to prove the existence of the limiting distribution. Their strategy cannot be applied directly to the sequence $\left\{q_{n}\right\}$ because the map $T$ does not have a finite invariant measure. However, it can be applied to the subsequence $\left\{\hat{q}_{n}\right\} \subset\left\{q_{n}\right\}$ and the Gauss-like map $R$. Thus we first prove the renewal-type Theorem [1.6] (see below) for the subsequence $\left\{\hat{q}_{n}\right\}$ and derive our Main Theorem from it.

Let us define the renewal time for the sequence $\left\{\hat{q}_{n}\right\}$ : given $L>0$, set

$$
\hat{n}_{L}=\hat{n}_{L}(\hat{\omega})=\hat{n}_{L}\left(\hat{\omega}^{+}\right):=\min \left\{n \in \mathbb{N}: \hat{q}_{n}>L\right\} .
$$

Theorem 1.6. For each $N_{1}, N_{2} \in \mathbb{N}$ there exists a probability measure $\mathrm{P}_{N_{1}, N_{2}}^{\prime}$ on $(1,+\infty) \times \Sigma^{N_{1}+N_{2}}$ such that for all $a, b>1, c_{j} \in \Sigma,-N_{1}<j \leq N_{2}$,

$$
\begin{array}{r}
\mu\left(\left\{\alpha: a<\frac{\hat{q}_{\hat{n}_{L}}}{L}<b, \sigma_{\hat{n}_{L}+j}=c_{j},-N_{1}<j \leq N_{2}\right\}\right) \stackrel{L \rightarrow \infty}{\longrightarrow} \\
\mathrm{P}_{N_{1}, N_{2}}^{\prime}\left((a, b) \times\left\{c_{-N_{1}+1}\right\} \times \cdots \times\left\{c_{0}\right\} \times \cdots \times\left\{c_{N_{2}}\right\}\right) .
\end{array}
$$

The proofs of Theorem 1.6 and Main Theorem are given in Section 4 .

\subsection{Cylinders}

For $c_{i} \in \Sigma, i=1, \ldots, n$, define the cylinder of length $n$

$$
\mathcal{C}\left[c_{1}, \ldots, c_{n}\right]=\left\{\hat{\omega}^{+}=\left\{\sigma_{j}\right\}_{j \in \mathbb{N}} \in \Sigma^{\mathbb{N}}: \sigma_{i}=c_{i}, 1 \leq i \leq n\right\}
$$

and denote by $\mathfrak{C}_{n}^{+}$the set of all cylinders of length $n$. If $\pi: D(\hat{R})=\Sigma^{\mathbb{Z}} \rightarrow \Sigma^{\mathbb{N}}=(0,1] \backslash \mathbb{Q}$ is the natural projection and $\mathcal{C} \in \mathfrak{C}_{n}^{+}$, then we shall denote by $\hat{\mathcal{C}}$ the set $\pi^{-1} \mathcal{C} \subseteq D(\hat{R})$. More generally, given $c_{i} \in \Sigma,-n_{1} \leq i \leq n_{2}, n_{1,2} \in \mathbb{N}$, set

$$
\mathcal{C}\left[c_{-n_{1}}, \ldots, c_{0} ; c_{1}, \ldots, c_{n_{2}}\right]=\left\{\left\{\sigma_{j}\right\}_{j \in \mathbb{Z}} \in D(\hat{R}): \sigma_{i}=c_{i},-n_{1} \leq i \leq n_{2}\right\}
$$

and denote by $\mathfrak{C}_{n_{1}, n_{2}}$ the set of all such bi-sided cylinders.

Remark 1.7. Notice that $\mathcal{C}\left[m^{ \pm}\right]=\mathcal{C}\left[0 \cdot m^{ \pm}\right]=B(m, \pm 1)$. Moreover, after writing explicitly all cylinders of length one and integrating the density $f$, for all $h \cdot m^{ \pm} \in \Sigma$, we get

$$
\mu\left(\mathcal{C}\left[h \cdot m^{ \pm}\right]\right) \leq \frac{3}{\log 3\left(4 h^{2}+8 h+3\right)} \frac{1}{m^{2}} .
$$

In particular, the measures of our cylinders of length one are $\mathcal{O}\left(\frac{1}{m^{2}}\right)$ as $m \rightarrow \infty$, where the constants implied by the $\mathcal{O}$-notation depend on $h$ as above. 


\subsection{Special Flows}

Consider a probability space $(D, \mathcal{B}, \mu)$, an invertible $\mu$-preserving map $F: D \rightarrow D$ (the "base" transformation) and a positive function $\varphi: D \rightarrow \mathbb{R}^{+}$(the "roof" function) such that $I=\int_{D} \varphi(\omega) \mathrm{d} \mu(\omega)<\infty$. Define $D_{\Phi}=\{(x, y) \in D \times \mathbb{R}: 0 \leq y<\varphi(x)\} \subseteq D \times \mathbb{R}$ and set $\mu_{\Phi}$ as the normalized measure obtained by restriction of the product measure $I^{-1} \mu \times \lambda$ to $D_{\Phi}$, where $\lambda$ is the Lebesgue measure on $\mathbb{R}$. The special flow $\left\{\Phi_{t}\right\}_{t \in \mathbb{R}}$ built over $F$ under the roof function $\varphi$ is the one-parameter group of $\mu_{\Phi}$-preserving transformations on $D_{\Phi}$ whose action is defined as follows (see e.g. [5]):

$$
\left\{\begin{array}{l}
\Phi_{t}(x, y)=(x, y+t), \quad \text { if } 0 \leq y+t<\varphi(x) \\
\Phi_{\varphi(x)}(x, 0)=(F(x), 0) .
\end{array}\right.
$$

The flow moves a point $(x, y) \in D_{\Phi}$ vertically upward with unit speed to the "roof" point $(x, \varphi(x))$. After that, the point jumps to the "base" point $(F(x), 0)$ and continues moving vertically until the next jump and so on. We shall denote by

$$
S_{0}(\varphi, F)(x):=0, \quad S_{r}(\varphi, F)(x):=\sum_{i=0}^{r-1} \varphi\left(F^{i}(x)\right), \quad x \in D, r \in \mathbb{N}
$$

the $r$-th (non-normalized) Birkhoff sum of $\varphi$ along the trajectory of $x$ under $F$. Given $x \in D$ and $t \in \mathbb{R}^{+}$we define $r(x, t) \in \mathbb{N}$,

$$
r(x, t):=\min \left\{r \in \mathbb{N}: S_{r}(\varphi, F)(x)>t\right\} .
$$

The non-negative integer $r(x, t)-1$ is the number of discrete iterations of $F$ which the point $(x, 0) \in D_{\Phi}$ undergoes before time $t$. The flow $\Phi_{t}$ defined above acts therefore for $t>0$ as

$$
\Phi_{t}(x, 0)=\left(F^{r(x, t)-1}(x), t-S_{r(x, t)-1}(\varphi, T)(x)\right),
$$

while for $t<0$ the action of the flow is defined using the inverse map.

\section{Recurrence Relations and Denominator Estimates}

Consider the Euclidean continued fraction expansion $\left[a_{1}, a_{2}, a_{3}, \ldots\right]$ of $\alpha \in(0,1] \backslash \mathbb{Q}$. We have the recurrence formulae

$$
P_{n}=a_{n} P_{n-1}+P_{n-2}, \quad Q_{n}=a_{n} Q_{n-1}+Q_{n-2}, \quad n \in \mathbb{N},
$$

with $Q_{-1}=P_{0}=0, P_{-1}=Q_{0}=1$ and two well-known estimates on the quality of the approximation and on the growth of the denominators, [10]:

$$
\left|x-\frac{P_{n}}{Q_{n}}\right| \leq \frac{1}{Q_{n}^{2}}, \quad Q_{n} \geq 2^{\frac{n-1}{2}}, \quad n \in \mathbb{N}
$$

Similar recurrence formulae are valid for ECF-expansions (see [11]): for $\alpha=\left[\left[\left(k_{1}, \xi_{1}\right),\left(k_{2}, \xi_{2}\right), \ldots\right]\right]$ we have

$$
p_{n}=2 k_{n} p_{n-1}+\xi_{n-1} p_{n-2}, \quad q_{n}=2 k_{n} q_{n-1}+\xi_{n-1} q_{n-2}, \quad n \in \mathbb{N},
$$


with $q_{-1}=p_{0}=0, p_{-1}=q_{0}=\xi_{0}=1$. However, estimates analogous to (5) are not available for ECF-expansions. We have indeed the weaker estimates (see [11] and [14])

$$
\left|x-\frac{p_{n}}{q_{n}}\right| \leq \frac{1}{q_{n}}, \quad q_{n} \geq n+1, \quad n \in \mathbb{N}
$$

and the latter, in particular, is optimal and cannot be improved. Nevertheless, we claim that the sequence $\left\{\hat{q}_{n}\right\}_{n}$ grows at least exponentially fast. Indeed we prove the following Lemma which provides an estimate similar to (51).

Lemma 2.1 (Growth of $R$-denominators, lower bound). For any $\hat{\omega} \in D(\hat{R})$, the denominators $\hat{q}_{n}=\hat{q}_{n}(\hat{\omega})=\hat{q}_{n}\left(\hat{\omega}^{+}\right)$satisfy the estimate

$$
\hat{q}_{n} \geq 3^{\frac{n}{3}}, \quad n \in \mathbb{N} .
$$

Proof. Recall that $\nu_{n}=\nu_{n-1}+1+\tau\left(R^{n-1}\left(\hat{\omega}^{+}\right)\right)$for $n \in \mathbb{N}$. Using (16) one can see that $\hat{q}_{n}=q_{\nu_{n}} \geq$ $3 q_{\nu_{n-1}-2}$. In particular we get for any $n \geq 4$

$$
\hat{q}_{n}=q_{\nu_{n}} \geq 3 q_{\nu_{n-1}-2} \geq 3 q_{\nu_{n-3}}=3 \hat{q}_{n-3},
$$

where the second inequality follows from the monotonicity of the sequence $\left\{q_{n}\right\}$ because $\nu_{n-1}-$ $\nu_{n-3} \geq 2$. Now, by the second inequality of (17), we have

$$
\hat{q}_{i} \geq q_{i+1} \geq i+2 \quad \text { for } i=1,2,3
$$

and therefore from (9) we get the estimate for $n \in \mathbb{N}$

$$
\hat{q}_{n}=q_{\nu_{n}} \geq\left([n-1]_{3}+3\right) \cdot 3^{\left(\left\lceil\frac{n}{3}\right\rceil-1\right)} \geq 3^{\left\lceil\frac{n}{3}\right\rceil} \geq 3^{\frac{n}{3}},
$$

where $[p]_{3}=p(\bmod 3)$ and $\lceil p\rceil:=\min \{m \in \mathbb{N}: m \geq p\}$. This concludes the proof of the Lemma.

Remark 2.2. Our proof actually gives $\hat{q}_{n} \geq \max \left\{n+2,3^{\frac{n}{3}}\right\}, n \in \mathbb{N}$, which can be replaced by (8) for $n \geq 6$. However, (8) will be enough for our purposes.

The following Lemma provides an upper bound for the growth of the $R$-denominators $\hat{q}_{n}$, proving that typically (i.e. $\mu$-almost surely) they grow at most exponentially fast. The proof is analogous to the one given by Khinchin [10] for the Euclidean continued fraction expansions.

Lemma 2.3 (Growth of $R$-denominators, upper bound). There exists a constant $C_{1}>0$ such that for $\mu$-almost every $\hat{\omega}^{+} \in(0,1) \backslash \mathbb{Q}=\Sigma^{\mathbb{N}}$ the denominators $\hat{q}_{n}=\hat{q}_{n}\left(\hat{\omega}^{+}\right)$satisfy the estimate

$$
\hat{q}_{n} \leq e^{C_{1} n}
$$

for all sufficiently large $n$. 
Proof. Let $\hat{\omega}^{+}=\left(h_{1} \cdot m_{1}^{ \pm}, h_{2} \cdot m_{2}^{ \pm}, \ldots\right) \in \Sigma^{\mathbb{N}}$. By the definition of $\nu_{n}$ we get

$$
\hat{q}_{n}=q_{\nu_{n}}=q_{h_{1}+\ldots+h_{n}+n+1} \leq q_{h_{1}+\ldots+h_{n+1}+n+1}=q_{\nu_{n+1}-1} .
$$

Now, using (6) one can show that $\frac{1}{2} m_{n}\left(h_{n}+1\right) q_{\nu_{n-1}-1} \leq q_{\nu_{n}-1} \leq 6 m_{n}\left(h_{n}+1\right) q_{\nu_{n-1}-1}$ and therefore $\frac{1}{2^{n}} m_{n}\left(h_{n}+1\right) \cdots m_{1}\left(h_{1}+1\right) \leq q_{\nu_{n}-1} \leq 6^{n} m_{n}\left(h_{n}+1\right) \cdots m_{1}\left(h_{1}+1\right)$. Defining $a_{2 j-1}:=m_{j}$ and $a_{2 j}:=\left(h_{j}+1\right)$ for $j \in \mathbb{N}$, the previous inequalities become

$$
\frac{1}{2^{n}} \prod_{j=1}^{2 n} a_{j} \leq q_{\nu_{n}-1} \leq 6^{n} \prod_{j=1}^{2 n} a_{j}
$$

Let us show that the product $\prod_{j=1}^{2 n} a_{j}$ is bounded by $e^{A n}$ Lebesgue-almost surely for some $A>0$. For $s \geq 1$ define $E_{n}(s):=\left\{\hat{\omega}^{+} \in \Sigma^{\mathbb{N}}: \prod_{j=1}^{2 n} a_{j} \geq s\right\}$. This set can be written as union of intervals of the form $J_{n}=\left\{\hat{\omega}^{+} \in \Sigma^{\mathbb{N}}:\left(\left(\hat{\omega}^{+}\right)_{j}\right)_{j=1}^{n}=\left(h_{1} \cdot m_{1}^{ \pm}, \ldots, h_{n} \cdot m_{n}^{ \pm}\right)\right\}$and each of these intervals has length $\left|J_{n}\right| \leq \frac{4}{3} \frac{1}{q_{\nu_{n}-1}^{2}}$. Thus, by the first inequality of (12), $\left|J_{n}\right| \leq \frac{4}{3} 2^{2 n} \prod_{j=1}^{2 n} \frac{1}{a_{j}^{2}}$. Reasoning as in [10] (§III.14) we obtain

$$
\left|E_{n}(s)\right|<\frac{4}{3} 2^{2 n} \cdot \sum_{\substack{a_{1}, \ldots, a_{2 n} \in \mathbb{N} \\ a_{1} \ldots a_{2 n} \geq s}} \prod_{j=1}^{2 n} \frac{1}{a_{j}^{2}}<\frac{4}{3} \cdot \frac{2^{4 n}}{s} \sum_{j=0}^{2 n-1} \frac{(\log s)^{j}}{j !} .
$$

In particular for $s=e^{A n}$ by Stirling's formula one gets

$$
\left|E_{n}\left(e^{A n}\right)\right|<\frac{4}{3} e^{n(4 \log 2-A)}(2 n) \frac{(A n)^{2 n}}{(2 n) !} 2^{2 n} \leq C_{2} \sqrt{n} e^{-n(A-2 \log A-2 \log 2-2)},
$$

for some $C_{2}>0$. Choosing $A$ so that $(A-2 \log A-2 \log 2-2)>0$ we give an upper bound for $\left|E_{n}\left(e^{A n}\right)\right|$ by the $n$-th term of a convergent series. Thus we get $\sum_{n=1}^{\infty}\left|E_{n}\left(e^{A n}\right)\right|<\infty$ and therefore Lebesgue-almost every $\hat{\omega}^{+} \in(0,1)$ belongs only to a finite number of $E_{n}\left(e^{A n}\right)$ 's. In other words, for sufficiently large $n, \prod_{j=1}^{2 n} a_{j} \leq e^{A n}$ Lebesgue-almost surely. Now, by (11) and (12) we get

$$
\hat{q}_{n} \leq q_{\nu_{n+1}-1} \leq 6^{n+1} \prod_{j=1}^{2 n+2} a_{j} \leq 6^{n+1} e^{A(n+1)} \leq e^{C_{1} n}
$$

for some $C_{1}>0$ for Lebesgue-almost every $\hat{\omega}^{+} \in \Sigma^{\mathbb{N}}$ and for all sufficiently large $n$. The assertion of the Lemma follows now from the absolute continuity of $\mu$ w.r.t. the Lebesgue measure on $(0,1]$.

\section{Reduction to a Special Flow}

\subsection{Roof function}

For $\hat{\omega}=\left(\ldots, \omega_{-1}, \omega_{0} ; \omega_{1}, \omega_{2}, \ldots\right)$, with $\omega_{i}=\left(k_{i}, \xi_{i}\right) \in \Omega, i \in \mathbb{Z}$, we define

$$
\psi(\hat{\omega}):=\sum_{i=2}^{\nu_{1}(\hat{\omega})} \log \left(\frac{\xi_{i}(\hat{\omega})}{\left(\hat{T}^{i} \hat{\omega}\right)^{-}}\right)=\sum_{i=2}^{\nu_{1}(\hat{\omega})} \log \left(2 k_{i}+\frac{\xi_{i-1}}{2 k_{i-1}+\frac{\xi_{i-2}}{2 k_{i-2}+\frac{\xi_{i-3}}{\ddots}}}\right)
$$


as roof function over $\hat{R}$. The reason for this definition will be clear from Lemma 3.5 ,

Remark 3.1. Recall that $\nu_{0}(\hat{\omega})=1$ for every $\hat{\omega} \in D(\hat{R})$ and that $\nu_{i}\left(\hat{R}^{j} \omega\right)=\nu_{i+j}(\hat{\omega})$ for every $i, j \geq 0$. Hence we have

$$
\psi\left(\hat{R}^{j} \hat{\omega}\right)=\sum_{i=\nu_{0}\left(\hat{R}^{j} \hat{\omega}\right)+1}^{\nu_{1}\left(\hat{R}^{j} \hat{\omega}\right)} \log \left(\frac{\xi_{i}\left(\hat{R}^{j} \hat{\omega}\right)}{\left(\hat{T}^{i} \hat{R}^{j} \hat{\omega}\right)^{-}}\right)=\sum_{i=\nu_{j}(\hat{\omega})+1}^{\nu_{j+1}(\hat{\omega})} \log \left(\frac{\xi_{i}(\hat{\omega})}{\left(\hat{T}^{i} \hat{\omega}\right)^{-}}\right) .
$$

In order to construct the special flow as described in Section 1.8, we have to check that our roof function is integrable.

Lemma 3.2. The roof function $\psi: D(\hat{R}) \rightarrow \mathbb{R}^{+}$is $\hat{\mu}$-integrable.

Proof. Let us denote $\tau=\tau(\hat{\omega})=\tau\left(\hat{\omega}^{+}\right)$and recall that $\nu_{1}(\hat{\omega})=\tau+2$. We can write $\psi=\psi_{0}+\psi_{1}+\psi_{2}$, where $\psi_{0,1,2}: D(\hat{R}) \rightarrow \mathbb{R}_{\geq 0}$,

$$
\begin{aligned}
& \psi_{0}(\hat{\omega})=\left\{\begin{array}{cl}
\sum_{i=2}^{\tau} \log \left(\frac{\xi_{i}(\hat{\omega})}{\left(\hat{T}^{i} \hat{\omega}\right)^{-}}\right) & \text {if } \tau \geq 2, \quad \text { and } \\
0 & \text { otherwise. }
\end{array}\right. \\
& \psi_{j}(\hat{\omega})=\left\{\begin{array}{cl}
\log \left(\frac{\xi_{\tau+j}(\hat{\omega})}{\left(\hat{T}^{\tau+j} \hat{\omega}\right)^{-}}\right) & \text {if }(j=1 \text { and } \tau \geq 1) \text { or } j=2, \\
0 & \text { if } j=1 \text { and } \tau=0 .
\end{array}\right.
\end{aligned}
$$

Let $-\frac{1}{3}<x<1$ such that $x=\left[\left[\left(0, \xi_{0}\right) ;\left(k_{0}, \xi_{-1}\right),\left(k_{-1}, \xi_{-2}\right), \ldots\right]\right]$. If $\tau \geq 2$, we have, for $i=2, \ldots, \tau$

$$
\frac{\xi_{i}(\hat{\omega})}{\left(\hat{T}^{i} \hat{\omega}\right)^{-}}=\frac{(i+1)-i x}{i-(i-1) x} \quad \text { and therefore } \quad \psi_{0}(\hat{\omega})=\log \left(\frac{(\tau+1)-\tau x}{2-x}\right)
$$

because the sum defining $\psi_{0}$ is telescopic. Using the fact that $\tau=h$ iff $\hat{\omega} \in \hat{\mathcal{C}}\left[h \cdot m^{ \pm}\right]$, the definition of the measure $\hat{\mu}$ and the estimates given in Remark 1.7 we get

$$
\begin{aligned}
\int_{0}^{1} \psi_{0}(\hat{\omega}) \mathrm{d} \hat{\mu}(\hat{\omega}) & =\sum_{\substack{h \cdot m^{ \pm} \in \Sigma \\
h \geq 2}} \int_{\hat{\mathcal{C}}\left[h \cdot m^{ \pm}\right]} \log \left(\frac{(h+1)-h x}{2-x}\right) \mathrm{d} \hat{\mu}(\hat{\omega}) \leq \\
& \leq \sum_{h \geq 2} \log (2 h+1) \sum_{m^{ \pm} \in \Omega^{*}} \mu\left(\mathcal{C}\left[h \cdot m^{ \pm}\right]\right) \leq \\
& \leq \frac{6}{\log 3} \sum_{h \geq 2} \frac{\log (2 h+1)}{4 h^{2}+8 h+3} \sum_{m \geq 1} \frac{1}{m^{2}} \leq 3 . \\
\int_{0}^{1} \psi_{1}(\hat{\omega}) \mathrm{d} \hat{\mu}(\hat{\omega}) & =\sum_{h \cdot m^{ \pm} \in \Sigma} \int_{\hat{\mathcal{C}}\left[h \cdot m^{ \pm}\right]} \log \left(2 m-\frac{h-(h-1) x}{(h+1)-h x}\right) \mathrm{d} \hat{\mu}(\hat{\omega}) \leq \\
\leq & \sum_{m^{ \pm} \in \Omega^{*}} \log (2 m) \sum_{h \geq 1} \mu\left(\mathcal{C}\left[h \cdot m^{ \pm}\right]\right) \leq \\
\leq & \frac{6}{\log 3} \sum_{m \geq 1} \frac{\log (2 m)}{m^{2}} \sum_{h \geq 1} \frac{1}{4 h^{2}+8 h+3} \leq 2 .
\end{aligned}
$$


Let us estimate the integral of $\psi_{2}$. Notice that $k_{1}(\hat{\omega})=m$ for $\hat{\omega} \in \hat{\mathcal{C}}\left[m^{ \pm}\right]$and $k_{1}(\hat{\omega})=1$ for $\hat{\omega} \in \hat{\mathcal{C}}\left[h \cdot m^{ \pm}\right]$with $h \in \mathbb{N}$. Moreover, $B(1,-1)=\left[\frac{1}{2}, 1\right]$ can be written as the disjoint union of $\mathcal{C}\left[h \cdot m^{ \pm}\right], h \in \mathbb{N}, m \in \Omega^{*}$, and its $\mu$-measure is $\mu(B(1,-1))=\log \frac{5}{3}$. Now, using also the $\hat{R}$-invariance of the measure $\hat{\mu}$ we get

$$
\begin{aligned}
\int_{0}^{1} \psi_{2}(\hat{\omega}) \mathrm{d} \hat{\mu}(\hat{\omega}) & \leq \int_{0}^{1} \log \left(2 k_{\nu_{1}}+1\right) \mathrm{d} \hat{\mu}(\hat{\omega})=\int_{0}^{1} \log \left(2 k_{1}+1\right) \mathrm{d} \hat{\mu}(\hat{\omega}) \leq \\
& \leq \frac{6}{\log 3} \sum_{m \geq 1} \frac{\log (2 m+1)}{m^{2}}+\log 3 \cdot \mu(B(1,-1)) \leq 15
\end{aligned}
$$

and this concludes the proof of the Lemma.

Lemma 3.3. Let $\mathcal{C} \in \mathfrak{C}_{n_{1}, n_{2}}$, with $n_{1} \in \mathbb{N}$ and $n_{2} \geq 2$, be any cylinder. Then there exist $\delta=\delta(\mathcal{C})>$ 0 and $M=M(\mathcal{C})>0$ such that

$$
\inf _{\hat{\omega} \in \mathcal{C}} \psi(\hat{\omega}) \geq \delta \quad \text { and } \quad \sup _{\hat{\omega} \in \mathcal{C}} \psi(\hat{\omega}) \leq M .
$$

Proof. Using the same notations introduced in the proof of Lemma 3.2, the statement of Lemma 3.3 follows easily from the following elementary estimates for $\psi_{1,2,3}$ on cylinders in $\mathfrak{C}_{2}^{+}$.

If $\hat{\omega} \in \mathcal{C}\left[0 \cdot m_{1}^{ \pm}, 0 \cdot m_{2}^{ \pm}\right]$, then $\psi_{0}(\hat{\omega})=\psi_{1}(\hat{\omega})=0$ and $0<\log \frac{9}{5} \leq \log \left(\frac{10 m_{2}-1}{5}\right) \leq \psi_{2}(\hat{\omega}) \leq$ $\log \left(2 m_{2}+1\right)$.

If $\hat{\omega} \in \mathcal{C}\left[0 \cdot m_{1}^{ \pm}, h_{2} \cdot m_{2}^{ \pm}\right], h_{2} \geq 1$, then $\psi_{0}(\hat{\omega})=\psi_{1}(\hat{\omega})=0$ and $0<\log \frac{9}{5} \leq \psi_{2}(\hat{\omega}) \leq \log 3$.

If $\hat{\omega} \in \mathcal{C}\left[h_{1} \cdot m_{1}^{ \pm}, 0 \cdot m_{2}^{ \pm}\right], h_{1} \geq 1$, then $0 \leq \psi_{0}(\hat{\omega}) \leq \log \left(2 h_{1}+1\right), 0 \leq \psi_{1}(\hat{\omega}) \leq \log \left(2 m_{1}\right)$ and $0<\log \frac{5}{3} \leq \log \left(\frac{6 m_{2}-1}{3}\right) \leq \psi_{2}(\hat{\omega}) \leq \log \left(2 m_{2}+1\right)$.

If $\hat{\omega} \in \mathcal{C}\left[h_{1} \cdot m_{1}^{ \pm}, h_{2} \cdot m_{2}^{ \pm}\right], h_{1,2} \geq 1$, then $0 \leq \psi_{0}(\hat{\omega}) \leq \log \left(2 h_{1}+1\right), 0 \leq \psi_{1}(\hat{\omega}) \leq \log \left(2 m_{1}\right)$ and $0<\log \frac{5}{3} \leq \psi_{2}(\hat{\omega}) \leq \log 3$.

\subsection{Mixing of the Special Flow}

Let us consider the special flow $\left\{\Phi_{t}\right\}_{t \in \mathbb{R}}$ built over $\hat{R}$ under the roof function $\psi$. Set $I=\int_{\mathcal{X}} \psi \mathrm{d} \hat{\mu}$ (it is finite by Lemma 3.2) and let $\tilde{\mu}=I^{-1} \hat{\mu} \times \lambda$ be the $\Phi_{t}$-invariant probability measure on $D_{\Phi}$. Recall that $\left\{\Phi_{t}\right\}_{t \in \mathbb{R}}$ is said to be mixing if, for all Borel subsets A and B of $D_{\Phi}$, we have

$$
\lim _{t \rightarrow \infty} \tilde{\mu}\left(\Phi_{-t}(\mathrm{~A}) \cap \mathrm{B}\right)=\tilde{\mu}(\mathrm{A}) \tilde{\mu}(\mathrm{B}) .
$$

Proposition 3.4. The flow $\left\{\Phi_{t}\right\}_{t \in \mathbb{R}}$ is mixing.

Proposition 3.4 is proven in Appendix A.

\subsection{Approximation by Birkhoff sums}

The following Lemma shows that $\log \hat{q}_{n}(\hat{\omega})$ can be approximated by a Birkhoff sum of the function $\psi$ along the trajectory of $\hat{\omega}$ under $\hat{R}$. Moreover this approximation is uniform in $\hat{\omega}$ and exponentially accurate as $n \rightarrow \infty$. Define

$$
g_{n}(\hat{\omega}):=\log \hat{q}_{n}(\hat{\omega})-S_{n}(\psi, \hat{R})(\hat{\omega}), \quad n \geq 0 .
$$


Lemma 3.5. There exists a function $g$ on $D(\hat{R})$ such that $g_{n}$ converges to $g$ uniformly in $\hat{\omega}$ and exponentially fast in $n$, i.e.

$$
\log \hat{q}_{n}(\hat{\omega})=S_{n}(\psi, \hat{R})(\hat{\omega})+g(\hat{\omega})+\varepsilon_{n}(\hat{\omega}), \quad \sup _{\hat{\omega} \in D(\hat{R})}\left|\varepsilon_{n}(\hat{\omega})\right|=\mathcal{O}\left(3^{-\frac{n}{3}}\right) .
$$

More precisely: for $n \geq 4$ we have $\sup _{\hat{\omega} \in D(\hat{R})}\left|\varepsilon_{n}(\hat{\omega})\right| \leq C_{3} 3^{-\frac{n}{3}}$, for some $C_{3}>0$.

Proof. Let $r_{n}=\frac{q_{n}}{q_{n-1}}, n \in \mathbb{N}$. Since $q_{0}=1$ we have $q_{n}=\prod_{i=1}^{n} r_{i}$. From (6) we get

$$
r_{i}=2 k_{i}+\frac{\xi_{i-1}}{r_{i-1}}=2 k_{i}+\frac{\xi_{i-1}}{2 k_{i-1}+\frac{\xi_{i-2}}{2 k_{i-2}+\ldots+\frac{\xi_{2}}{2 k_{2}+\frac{\xi_{1}}{2 k_{1}}}}}=\left[\left[\left(k_{i}, \xi_{i-1}\right),\left(k_{i-1}, \xi_{i-2}\right), \ldots,\left(k_{2}, \xi_{1}\right),\left(k_{1}, *\right)\right]\right]^{-1}
$$

and hence

$$
\log \hat{q}_{n}=-\sum_{i=1}^{\nu_{n}(\hat{\omega})} \log \left[\left[\left(k_{i}, \xi_{i-1}\right),\left(k_{i-1}, \xi_{i-2}\right), \ldots,\left(k_{2}, \xi_{1}\right),\left(k_{1}, *\right)\right]\right] .
$$

From (14) we get

$$
S_{n}(\psi, \hat{R})(\hat{\omega})=\sum_{j=0}^{n-1} \psi\left(\hat{R}^{j} \hat{\omega}\right)=\sum_{j=0}^{n-1}\left(\sum_{i=\nu_{j}(\hat{\omega})+1}^{\nu_{j+1}(\hat{\omega})} \log \left(\frac{\xi_{i}(\hat{\omega})}{\left(\hat{T}^{i} \hat{\omega}\right)^{-}}\right)\right) .
$$

From the definition (15) and (17,[18) we obtain

$$
\left(g_{n+1}-g_{n}\right)(\hat{\omega})=\sum_{i=\nu_{n}(\hat{\omega})+1}^{\nu_{n+1}(\hat{\omega})} \log r_{i}(\hat{\omega})-\psi\left(\hat{R}^{n} \hat{\omega}\right)=\sum_{i=\nu_{n}(\hat{\omega})+1}^{\nu_{n+1}(\hat{\omega})} \log \left(r_{i}(\hat{\omega}) \cdot \frac{\left(\hat{T}^{i} \hat{\omega}\right)^{-}}{\xi_{i}(\hat{\omega})}\right) .
$$

Our goal is to prove that the sequence $\left\{g_{n}\right\}_{n}$ converges exponentially fast. In order to show this, we will estimate $\left|g_{n+1}-g_{n}\right|$ by estimating each term of the sum in (19) for $n \geq 4$.

Denoting $\tau=\tau\left(R^{n}\left(\hat{\omega}^{+}\right)\right)$and $j=\nu_{n}(\hat{\omega})$, for $i=j+l$ and $1 \leq l \leq \tau-1$ we have

$$
\begin{aligned}
& \log \left(r_{i}(\hat{\omega}) \cdot \frac{\left(\hat{T}^{i} \hat{\omega}\right)^{-}}{\xi_{i}(\hat{\omega})}\right)=\log \left(\frac{\left.2-\frac{1}{2-\frac{1}{2-\ldots-\frac{1}{2-\frac{1}{2-\beta}}}} \text { ("2" appears } l \text { times }\right)}{\left.2-\frac{1}{2-\frac{1}{2-\ldots-\frac{1}{2-\frac{1}{2-\gamma}}}} \text { ("2" appears } l \text { times }\right)}\right)= \\
& =\log \left(\frac{(l+1)-l \beta}{(l+1)-l \gamma} \cdot \frac{l-(l-1) \gamma}{l-(l-1) \beta}\right)=\log (1+\zeta)-\log (1+\eta)
\end{aligned}
$$

where

$$
\begin{gathered}
\beta=\left[\left[\left(k_{j}, \xi_{j-1}\right),\left(k_{j-1}, \xi_{j-2}\right), \ldots,\left(k_{2}, \xi_{1}\right),\left(k_{1}, *\right)\right]\right], \\
\gamma=\left[\left[\left(k_{j}, \xi_{j-1}\right),\left(k_{j-1}, \xi_{j-2}\right), \ldots,\left(k_{1}, \xi_{0}\right),\left(k_{0}, \xi_{-1}\right), \ldots\right]\right], \\
\zeta=\frac{l(\gamma-\beta)}{(l+1)-l \gamma} \quad \text { and } \quad \eta=\frac{(l-1)(\gamma-\beta)}{l-(l-1) \gamma} .
\end{gathered}
$$


It is easy to see that $\frac{1}{3} \leq \gamma \leq \frac{3}{5}$ and from this we get

$$
|\zeta-\eta|=\frac{|\gamma-\beta|}{((l+1)-l \gamma)(l-(l-1) \gamma)} \leq \frac{25}{4 l^{2}+16 l+15}|\gamma-\beta|
$$

Now we want to estimate $|\gamma-\beta|$ using (8), i.e. in terms of $R$-convergents of $\gamma$. From its definition we know that $\beta$ is the $j$-th $T$-convergent of $\gamma$ but in general it is not a $R$-convergent. However it can be shown that $j \geq \nu_{n-1}(\gamma)$ and therefore $q_{j}(\gamma) \geq q_{\nu_{n-1}(\gamma)}(\gamma)=\hat{q}_{n-1}(\gamma)$. This, along with (778), leads us to $|\gamma-\beta| \leq \frac{1}{\hat{q}_{n-1}(\gamma)} \leq 3^{\frac{1-n}{3}}$ and we choose $n \geq 4$ in order to guarantee that $\zeta, \eta>-1$. Now by (20,22) we get

$$
\left|\log \left(r_{i}(\hat{\omega}) \cdot \frac{\left(\hat{T}^{i} \hat{\omega}\right)^{-}}{\xi_{i}(\hat{\omega})}\right)\right| \leq \frac{C_{4}}{4 l^{2}+16 l+15} \cdot 3^{\frac{1-n}{3}}
$$

for some constant $C_{4}>0$. Now, let us estimate the last two terms in the sum in (19), i.e. $i=j+\tau$ and $i=j+\tau+1$. We introduce $\beta^{\prime}=\left[\left[\left(k_{i}, \xi_{i-1}\right),\left(k_{i-1}, \xi_{i-2}\right), \ldots,\left(k_{2}, \xi_{1}\right),\left(k_{1}, *\right)\right]\right]$ and $\gamma^{\prime}=\left[\left[\left(k_{i}, \xi_{i-1}\right),\left(k_{i-1}, \xi_{i-2}\right), \ldots,\left(k_{1}, \xi_{0}\right),\left(k_{0}, \xi_{-1}\right), \ldots\right]\right]$ and we observe that, since $\beta^{\prime}$ is the $i$-th $T$ convergent of $\gamma^{\prime}$, from (7) we get that $\left|\gamma^{\prime}-\beta^{\prime}\right| \leq \frac{1}{q_{i}} \leq \frac{1}{i+1} \leq \frac{1}{2}$. This estimate and the fact that $2 k_{i}-\gamma^{\prime} \geq 1$ give us

$$
\begin{gathered}
\log \left(r_{i}(\hat{\omega}) \cdot \frac{\left(\hat{T}^{i} \hat{\omega}\right)^{-}}{\xi_{i}(\hat{\omega})}\right)=\log \left(\frac{2 k_{i}-\beta^{\prime}}{2 k_{i}-\gamma^{\prime}}\right)=\log \left(1+\frac{\gamma^{\prime}-\beta^{\prime}}{2 k_{i}-\gamma^{\prime}}\right) \quad \text { and } \\
\left|\log \left(1+\frac{\gamma^{\prime}-\beta^{\prime}}{2 k_{i}-\gamma^{\prime}}\right)\right| \leq 3\left|\gamma^{\prime}-\beta^{\prime}\right| .
\end{gathered}
$$

Reasoning as above we get $\left|\gamma^{\prime}-\beta^{\prime}\right| \leq 3^{\frac{1-n}{3}}$ when $n \geq 4$ for $i=j+\tau$ and $i=j+\tau+1$. Therefore

$$
\sum_{i=j+\tau}^{j+\tau+1}\left|\log \left(r_{i}(\hat{\omega}) \cdot \frac{\left(\hat{T}^{i} \hat{\omega}\right)^{-}}{\xi_{i}(\hat{\omega})}\right)\right| \leq 2 \cdot 3^{\frac{4-n}{3}} \leq 3^{\frac{7-n}{3}} .
$$

Now we estimate the sum in (19) using (23+24) and the convergence of $\sum_{l=1}^{\infty}\left(4 l^{2}+16 l+15\right)^{-1}$ :

$$
\left|g_{n+1}-g_{n}\right| \leq C_{4} \sum_{l=1}^{\tau-1} \frac{3^{\frac{1-n}{3}}}{4 l^{2}+16 l+15}+3^{\frac{7-n}{3}} \leq C_{5} 3^{-\frac{n}{3}}, \quad n \geq 4,
$$

for some $C_{5}>0$ and uniformly in $\hat{\omega}$. The latter estimate allows us to define

$$
g(\hat{\omega}):=\sum_{n=0}^{\infty}\left(g_{n+1}-g_{n}\right)(\hat{\omega})
$$

and for $n \geq 4$

$$
\sup _{\hat{\omega} \in D(\hat{R})}\left|\varepsilon_{n}(\hat{\omega})\right|=\sup _{\hat{\omega} \in D(\hat{R})}\left|g(\hat{\omega})-g_{n}(\hat{\omega})\right| \leq C_{5} \sum_{m=n}^{\infty} 3^{-\frac{m}{3}} \leq C_{6} 3^{-\frac{n}{3}},
$$

for some $C_{6}>0$. The Lemma is therefore proven setting $C_{3}=C_{6}$. 
The following Lemma shows that on each bi-sided cylinder of length $n$ (for sufficiently large $n$ ) the function $g$ defined in (25) can be approximated by a constant up to an error which is exponentially small with $n$.

Lemma 3.6. If $\hat{\omega}^{\prime}, \hat{\omega}^{\prime \prime} \in \mathcal{C}\left[c_{-n}, \ldots, c_{0} ; c_{1}, \ldots, c_{n}\right], c_{j} \in \Sigma$, and $n \geq 4$, then

$$
\left|g\left(\hat{\omega}^{\prime}\right)-g\left(\hat{\omega}^{\prime \prime}\right)\right| \leq C_{7} 3^{-\frac{n}{3}}
$$

where $C_{7}>0$ is an absolute constant.

Proof. By Lemma 3.5 we have

$$
\left|g\left(\hat{\omega}^{\prime}\right)-g\left(\hat{\omega}^{\prime \prime}\right)\right| \leq C_{6} 3^{-\frac{n}{3}}+\left|g_{n}\left(\hat{\omega}^{\prime}\right)-g_{n}\left(\hat{\omega}^{\prime \prime}\right)\right|,
$$

for every $n \geq 4$. We need to estimate the second term in the right hand side of (27). Let $\hat{\omega}^{\prime}=\left\{\left(k_{i}^{\prime}, \xi_{i}^{\prime}\right)\right\}_{i \in \mathbb{Z}}$ and $\hat{\omega}^{\prime \prime}=\left\{\left(k_{i}^{\prime \prime}, \xi_{i}^{\prime \prime}\right)\right\}_{i \in \mathbb{Z}}$ with $\left(k_{i}^{\prime}, \xi_{i}^{\prime}\right),\left(k_{i}^{\prime \prime}, \xi_{i}^{\prime \prime}\right) \in \Omega$. By assumption we have $\nu_{j}\left(\hat{\omega}^{\prime}\right)=\nu_{j}\left(\hat{\omega}^{\prime \prime}\right)=\nu_{j}$ for $-n-1 \leq j \leq n$ and $\left(k_{i}^{\prime}, \xi_{i}^{\prime}\right)=\left(k_{i}^{\prime \prime}, \xi_{i}^{\prime \prime}\right)=\left(k_{i}, \xi_{i}\right)$ for $\nu_{-n-1} \leq i \leq \nu_{n+1}-1$. From (18) we get

$$
S_{n}(\psi, \hat{R})\left(\hat{\omega}^{\prime}\right)-S_{n}(\psi, \hat{R})\left(\hat{\omega}^{\prime \prime}\right)=\sum_{j=0}^{n-1} \sum_{i=\nu_{j}+1}^{\nu_{j+1}} \log \left(\frac{\left(\hat{T}^{i} \hat{\omega}^{\prime \prime}\right)^{-}}{\left(\hat{T}^{i} \hat{\omega}^{\prime}\right)^{-}}\right)
$$

The estimate of the sum indexed by $i$ is now done with the same technique used in the proof of Lemma 3.5. Denoting $\tau=\tau\left(R^{j}\left(\left(\hat{\omega}^{\prime}\right)^{+}\right)\right), \nu=\nu_{j}\left(\hat{\omega}^{\prime}\right)$ and $\bar{\nu}=-\nu_{-n-1}(\hat{\omega})$, for $i=\nu+l$ and $1 \leq l \leq \tau-1$ we have

$$
\log \left(\frac{\left(\hat{T}^{i} \hat{\omega}^{\prime \prime}\right)^{-}}{\left(\hat{T}^{i} \hat{\omega}^{\prime}\right)^{-}}\right)=\log (1+\zeta)-\log (1+\eta)
$$

where $\zeta$ and $\eta$ are as in (21), with

$$
\begin{aligned}
\beta & =\left[\left[\left(k_{\nu}, \xi_{\nu-1}\right), \ldots,\left(k_{-\bar{\nu}+1}, \xi_{-\bar{\nu}}\right),\left(k_{-\bar{\nu}}, \xi_{-\bar{\nu}-1}^{\prime}\right),\left(k_{-\bar{\nu}-1}^{\prime}, \xi_{-\bar{\nu}-2}^{\prime}\right), \ldots\right]\right] \text { and } \\
\gamma & =\left[\left[\left(k_{\nu}, \xi_{\nu-1}\right), \ldots,\left(k_{-\bar{\nu}+1}, \xi_{-\bar{\nu}}\right),\left(k_{-\bar{\nu}}, \xi_{-\bar{\nu}-1}^{\prime \prime}\right),\left(k_{-\bar{\nu}-1}^{\prime \prime}, \xi_{-\bar{\nu}-2}^{\prime \prime}\right), \ldots\right]\right] .
\end{aligned}
$$

Since (22) still holds, we want to estimate $|\gamma-\beta|$. Our assumptions imply that $\beta$ and $\gamma$ share the same ECF-expansion up to the index $\nu+\bar{\nu}+1$ and it can be shown that $\nu+\bar{\nu}+1 \geq \nu_{n+j-1}(\beta)$. Therefore by (88) we get $|\gamma-\beta| \leq \frac{1}{q_{\nu+\bar{\nu}+1}(\beta)} \leq \frac{1}{\hat{q}_{n+j-1}(\beta)} \leq 3^{\frac{1-n-j}{3}}$. We choose $n \geq 4$ as before and by (28) and (22) we find, for $i=\nu+l$,

$$
\left|\log \left(\frac{\left(\hat{T}^{i} \hat{\omega}^{\prime \prime}\right)^{-}}{\left(\hat{T}^{i} \hat{\omega}^{\prime}\right)^{-}}\right)\right| \leq \frac{C_{8}}{4 l^{2}+16 l+15} 3^{\frac{1-n-j}{3}},
$$

for some constant $C_{8}>0$. The last two terms, corresponding to $i=\nu+\tau$ and $i=\nu+\tau+1$, are estimated in the same way, obtaining $\left|\log \left(\frac{\left(\hat{T}^{i} \hat{\omega}^{\prime \prime}\right)^{-}}{\left(\hat{T}^{i} \hat{\omega}^{\prime}\right)^{-}}\right)\right| \leq 3^{\frac{4-n-j}{3}}$. Therefore

$$
\sum_{i=\nu_{j}+1}^{\nu_{j+1}}\left|\log \left(\frac{\left(\hat{T}^{i} \hat{\omega}^{\prime \prime}\right)^{-}}{\left(\hat{T}^{i} \hat{\omega}^{\prime}\right)^{-}}\right)\right| \leq C_{8} \sum_{l=1}^{\tau-1} \frac{3^{\frac{1-n-j}{3}}}{4 l^{2}+16 l+15}+3^{\frac{7-n-j}{3}} \leq C_{9} 3^{\frac{1-n-j}{3}}
$$


for some $C_{9}>0$ and hence

$$
\left|S_{n}(\psi, \hat{R})\left(\hat{\omega}^{\prime}\right)-S_{n}(\psi, \hat{R})\left(\hat{\omega}^{\prime \prime}\right)\right| \leq C_{9} \sum_{j=0}^{n-1} 3^{\frac{1-n-j}{3}} \leq C_{10} 3^{-\frac{n}{3}}
$$

for some $C_{10}>0$. Now by (15), since $\hat{q}_{n}\left(\hat{\omega}^{\prime}\right)=\hat{q}_{n}\left(\hat{\omega}^{\prime \prime}\right)$, we get $\left|g_{n}\left(\hat{\omega}^{\prime}\right)-g_{n}\left(\hat{\omega}^{\prime \prime}\right)\right| \leq C_{10} 3^{-\frac{n}{3}}$ and therefore, setting $C_{7}=C_{6}+C_{10}$, we get (26) , as claimed.

\subsection{Comparing Renewal Times}

Given $\hat{\omega}$ and $L$, we want to choose $T$ as a function of $L$ in order to compare $\hat{n}_{L}(\hat{\omega})$ and $r(\hat{\omega}, T)$. Recall that $\hat{n}_{L}(\hat{\omega})$ is uniquely determined by

$$
\log \hat{q}_{\hat{n}_{L}(\hat{\omega})-1} \leq \log L<\log \hat{q}_{\hat{n}_{L}(\hat{\omega})}
$$

Using (15), we rewrite (29) as

$$
S_{\hat{n}_{L}(\hat{\omega})-1}(\psi)(\hat{\omega})+g_{\hat{n}_{L}(\hat{\omega})-1}(\hat{\omega}) \leq \log L<S_{\hat{n}_{L}(\hat{\omega})}(\psi)(\hat{\omega})+g_{\hat{n}_{L}(\hat{\omega})}(\hat{\omega})
$$

after dropping the dependence on $\hat{R}$ in the notation for the Birkhoff sums. To avoid the dependence of time $T$ on $\hat{\omega}$, let us consider a set $\mathcal{C} \subset D(\hat{R})$ and denote $g_{\mathcal{C}}:=\sup _{\hat{\omega} \in \mathcal{C}} g(\hat{\omega})$. Assume that all $\hat{\omega} \in \mathcal{C}$ satisfy $\left|g(\hat{\omega})-g_{\mathcal{C}}\right| \leq \frac{\varepsilon}{2}$. We shall deal with such sets in the proof Theorem 1.6.

The following Lemma guarantees that $\hat{n}_{L}(\hat{\omega})$ grows uniformily when $L$ grows for $\hat{\omega}$ belonging to a set of sufficiently large measure. This fact is not obvious because a priori the $R$-denominators $\hat{q}_{n}(\hat{\omega})$ might grow very fast for some $\hat{\omega}$ and for that reason $L \mapsto \hat{n}_{L}(\hat{\omega})$ might be very slowly increasing. However, using Lemma 2.3, we prove that this cannot happen on a set of large measure.

Lemma 3.7. For each measurable $\mathcal{C} \subset D(\hat{R})$ and $\varepsilon>0$, there exists a measurable set $\mathcal{C}^{\prime} \subseteq \mathcal{C}$ such that $\hat{\mu}\left(\mathcal{C} \backslash \mathcal{C}^{\prime}\right) \leq \varepsilon \hat{\mu}(\mathcal{C})$ and $\min _{\hat{\omega} \in \mathcal{C}^{\prime}} \hat{n}_{L}(\hat{\omega}) \rightarrow \infty$ uniformly as $L \rightarrow \infty$.

Likewise, given $\varepsilon>0$, there exists a measurable set $\mathcal{C}_{\varepsilon} \subseteq(0,1]$ such that $\mu\left((0,1] \backslash \mathcal{C}_{\varepsilon}\right) \leq \varepsilon$ and $\min _{\hat{\omega} \in \mathcal{C}_{\varepsilon}} \hat{n}_{L}(\hat{\omega}) \rightarrow \infty$ uniformly as $L \rightarrow \infty$.

Proof. Let us consider $\overline{\mathcal{C}}=\pi(\mathcal{C})$ where $\pi: D(\hat{R}) \rightarrow(0,1] \backslash \mathbb{Q}$ is the natural projection. By Lemma 2.3 we know that there exists a set $\overline{\mathcal{C}}_{1} \subseteq \overline{\mathcal{C}}$, with $\mu\left(\overline{\mathcal{C}} \backslash \overline{\mathcal{C}}_{1}\right)=0$, and $\bar{n} \in \mathbb{N}$ such that for every $n>\bar{n}$ and every $\hat{\omega}^{+} \in \overline{\mathcal{C}}_{1}$ we have $\frac{\log \hat{q}_{n}\left(\hat{\omega}^{+}\right)}{n} \leq C_{1}$. Moreover there exists a set $\overline{\mathcal{C}}_{2} \subseteq \overline{\mathcal{C}}$, with $\mu\left(\overline{\mathcal{C}} \backslash \overline{\mathcal{C}}_{2}\right) \leq \varepsilon \mu(\overline{\mathcal{C}})$ and a constant $C_{11}=C_{11}(\overline{\mathcal{C}}, \varepsilon)$ such that for every $\hat{\omega}^{+} \in \overline{\mathcal{C}}_{2}$ we have $\frac{\log \hat{q}_{j}\left(\hat{\omega}^{+}\right)}{j} \leq C_{11}$ for $j=1, \ldots, \bar{n}$. Setting $\overline{\mathcal{C}}^{\prime}=\overline{\mathcal{C}}_{1} \cap \overline{\mathcal{C}}_{2}$ and $C_{12}=C_{12}(\overline{\mathcal{C}}, \varepsilon)=\max \left\{C_{1}, C_{11}\right\}$ we have $\mu\left(\overline{\mathcal{C}} \backslash \overline{\mathcal{C}}^{\prime}\right) \leq \varepsilon \mu(\overline{\mathcal{C}})$ and $\hat{q}_{n} \leq C_{12} n$ for every $\hat{\omega}^{+} \in \overline{\mathcal{C}}^{\prime}$. Since by construction all the functions $\hat{q}_{n}(\cdot)$ and $\hat{n}_{L}(\cdot)$ are constant on the fibers $\pi^{-1} \alpha, \alpha \in(0,1] \backslash \mathbb{Q}$, setting $\mathcal{C}^{\prime}=\pi^{-1} \overline{\mathcal{C}}^{\prime}$, the same statement is true for all $\hat{\omega} \in \mathcal{C}^{\prime}$ and $\hat{\mu}\left(\mathcal{C} \backslash \mathcal{C}^{\prime}\right) \leq \varepsilon \hat{\mu}(\mathcal{C})$. By definition $\hat{q}_{\hat{n}_{L}(\hat{\omega})}>L$ and this implies that $\min _{\hat{\omega} \in \mathcal{C}^{\prime}}\left(\hat{n}_{L}(\hat{\omega})\right) \geq \frac{1}{C_{12}} \log \hat{q}_{\hat{n}_{L}(\hat{\omega})} \geq \frac{\log L}{C_{12}}$. This proves the first part of the Lemma. The second part is proven in the same way. 
The following Lemma considers a bi-sided cylinder $\mathcal{C}$ and shows that for a suitable choice of $T$ as a function of $L$ and $\mathcal{C}$, the two quantities $\hat{n}_{L}(\hat{\omega})$ and $r(\hat{\omega}, T)$ coincide on a subset of $\mathcal{C}$ with relatively large measure.

Lemma 3.8. Let $n \geq 4$ and $\varepsilon>0$. Let us consider $\mathcal{C} \in \mathfrak{C}_{n, n}$ and assume that $\left|g(\hat{\omega})-g_{\mathcal{C}}\right| \leq \frac{\varepsilon}{2}$ for all $\hat{\omega} \in \mathcal{C}$. Define $T=T(L, \mathcal{C}):=\log L-g_{\mathcal{C}}$ and $U=U(\mathcal{C}):=\left\{\hat{\omega} \in \mathcal{C}: \hat{n}_{L}(\hat{\omega}) \neq r(\hat{\omega}, T)\right\}$. Then there exists $L_{0}=L_{0}(\mathcal{C})>0$ such that, for all $L \geq L_{0}$, we have $\hat{\mu}(U) \leq 7 \varepsilon \hat{\mu}(\mathcal{C})$.

We shall provide only a sketch of the proof of Lemma 3.8, since it is similar to the proof of Lemma 3.4 in [18].

Sketch of the proof. Let $T=T(L, \mathcal{C})=\log L-g_{\mathcal{C}}$. By definition we have

$$
S_{r(\hat{\omega}, T)-1}(\psi)(\hat{\omega}) \leq T=\log L-g_{\mathcal{C}}<S_{r(\hat{\omega}, T)}(\psi)(\hat{\omega}) .
$$

Let $\mathcal{C}^{\prime} \subseteq \mathcal{C}$ be as in Lemma 3.7 and define two sets $U_{ \pm \varepsilon} \subset D(\hat{R})$ as

$$
U_{-\varepsilon}:=\left\{\hat{\omega}: T<S_{r(\hat{\omega}, T)}(\psi)(\hat{\omega}) \leq T+\varepsilon\right\} \quad \text { and } \quad U_{\varepsilon}:=\left\{\hat{\omega}: T-\varepsilon<S_{r(\hat{\omega}, T)-1}(\psi)(\hat{\omega}) \leq T\right\} .
$$

Using (30-31) and Lemmata 3.5 and 3.7 (we are assuming $n \geq 4$ ) it is possible to show that for some $L_{0}>0$ and every $L \geq L_{0}$ we have

$$
U \cap \mathcal{C}^{\prime} \subseteq\left(U_{\varepsilon} \cup U_{-\varepsilon}\right) \cap \mathcal{C}^{\prime}
$$

According to the definition (4) of the special flow, the sets $U_{ \pm \varepsilon}$ can be rewritten as

$$
U_{\varepsilon}=\left\{(\hat{\omega}, 0): \Phi_{T}(\hat{\omega}, 0) \in D_{\Phi}^{\varepsilon}\right\} \quad \text { and } \quad U_{-\varepsilon}=\left\{(\hat{\omega}, 0): \Phi_{T}(\hat{\omega}, 0) \in D_{\Phi}^{-\varepsilon}\right\},
$$

where $D_{\Phi}^{\varepsilon}:=D(\hat{R}) \times[0, \varepsilon)$ and $D_{\Phi}^{-\varepsilon}:=\{(\hat{\omega}, y): \psi(\hat{\omega})-\varepsilon \leq y<\psi(\hat{\omega})\}$. Our aim now is to use mixing of $\left\{\Phi_{t}\right\}_{t \in \mathbb{R}}$ in order to estimate the measures of $U_{ \pm \varepsilon}$. Since our special flow is 3-dimensional, we need to "thicken" them as follows. Using Lemma 3.3 ( since $n \geq 4$ ) we can choose $0<\delta \leq \varepsilon$ such that $\delta<\min _{\hat{\omega} \in \mathcal{C}} \psi(\hat{\omega})$ and construct two subsets of $D_{\Phi}$ :

$$
U_{ \pm \varepsilon}^{\delta}:=\left\{(\hat{\omega}, z): 0 \leq z<\delta, \Phi_{T}(\hat{\omega}, z) \in D_{\Phi}^{ \pm \varepsilon}\right\}=D_{\Phi}^{\delta} \cap \Phi_{-T}\left(D_{\Phi}^{ \pm \varepsilon}\right) .
$$

Again using the definition (4) it is possible to show that $\left(U_{\varepsilon} \cap \mathcal{C}\right) \times[0, \delta) \subseteq U_{\varepsilon+\delta}^{\delta}$ and $\left(U_{-\varepsilon} \cap \mathcal{C}\right) \times$ $[0, \delta) \subseteq U_{-\varepsilon}^{\delta} \cup U_{\delta}^{\delta}$. Define now $\mathcal{C}_{\delta}^{\prime}:=\mathcal{C}^{\prime} \times[0, \delta)$. So far we proved

$$
\begin{aligned}
\left(U \cap \mathcal{C}^{\prime}\right) \times[0, \delta) & \subseteq\left(\left(U_{+\varepsilon} \cup U_{-\varepsilon}\right) \cap \mathcal{C}^{\prime}\right) \times[0, \delta) \subseteq \mathcal{C}_{\delta}^{\prime} \cap\left(U_{-\varepsilon}^{\delta} \cup U_{\delta+\varepsilon}^{\delta}\right)= \\
& =\mathcal{C}_{\delta}^{\prime} \cap \Phi_{-T}\left(D_{\Phi}^{-\varepsilon} \cup D_{\Phi}^{\delta+\varepsilon}\right) .
\end{aligned}
$$

From the previous inclusions and the mixing property of the special flow (Proposition 3.4), one can find some $T_{0}>0$ such that, for any $T \geq T_{0}$, we have

$$
\begin{aligned}
\hat{\mu}\left(U \cap \mathcal{C}^{\prime}\right) \cdot \delta & =\tilde{\mu}\left(\left(U \cap \mathcal{C}^{\prime}\right) \times[0, \delta)\right) \leq \tilde{\mu}\left(\mathcal{C}_{\delta}^{\prime} \cap \Phi_{-T}\left(D_{\Phi}^{-\varepsilon} \cup D_{\Phi}^{\delta+\varepsilon}\right)\right) \leq \\
& \leq 2 \hat{\mu}\left(\mathcal{C}^{\prime}\right) \cdot \delta \cdot \tilde{\mu}\left(D_{\Phi}^{-\varepsilon} \cup D_{\Phi}^{\delta+\varepsilon}\right) \leq 2 \hat{\mu}\left(\mathcal{C}^{\prime}\right) \cdot \delta \cdot 3 \varepsilon,
\end{aligned}
$$

where the last inequality follows from the estimate $\tilde{\mu}\left(D_{\Phi}^{ \pm \varepsilon}\right) \leq \varepsilon$. Therefore we get $\hat{\mu}\left(U \cap \mathcal{C}^{\prime}\right) \leq$ $6 \varepsilon \hat{\mu}\left(\mathcal{C}^{\prime}\right)$. We can enlarge $L_{0}$ so that $\log L_{0}-g_{\mathcal{C}} \geq T_{0}, L \geq L_{0}$ implies $T \geq T_{0}$ and (32) still holds. Thus, $\hat{\mu}(U) \leq \hat{\mu}\left(U \backslash \mathcal{C}^{\prime}\right)+6 \varepsilon \hat{\mu}(\mathcal{C}) \leq 7 \varepsilon \hat{\mu}(\mathcal{C})$ and the Lemma is proven. 


\section{Proof of the Existence of the Limiting Distribution}

Recall that for $L>0$ we defined $\hat{n}_{L}(\hat{\omega})=\min \left\{n \in \mathbb{N}: \hat{q}_{n}(\hat{\omega})>L\right\}$.

Proof of Theorem 1.6. Assume $1<a<b$ and $c_{j} \in \Sigma,-N_{1}<j \leq N_{2}$. Our aim is to estimate the expression in (3). Since the quantities $q_{i}(\hat{\omega})$ (and in particular $\hat{q}_{i}(\hat{\omega})$ ) and $\hat{n}_{L}(\hat{\omega})$ depend only on $\alpha=\hat{\omega}^{+}$, for $\hat{n}_{L}(\omega)>N_{1}$, we can rewrite the condition $\sigma_{\hat{n}_{L}+j}=c_{j},-N_{1}<j \leq N_{2}$, as

$$
\hat{R}^{\hat{n}_{L}(\hat{\omega})-1}(\hat{\omega}) \in \mathcal{C}_{N_{1}, N_{2}}, \quad \text { where } \mathcal{C}_{N_{1}, N_{2}}:=\hat{R}^{N_{1}-1}\left(\hat{\mathcal{C}}\left[c_{-N_{1}+1}, c_{-N_{1}+2}, \ldots, c_{0}, \ldots, c_{N_{2}}\right]\right) .
$$

Given two functions $F_{1}, F_{2}$ on $D(\hat{R})$, we define $D_{\Phi}\left(F_{1}, F_{2}\right):=\left\{(\hat{\omega}, y) \in D_{\Phi}: \psi(\hat{\omega})-F_{2}(\hat{\omega})<y<\right.$ $\left.\psi(\hat{\omega})-F_{1}(\hat{\omega})\right\}$. Notice that for some values of $F_{1}(\hat{\omega})$ and $F_{2}(\hat{\omega})$, the corresponding set of $y$ can be empty. Moreover, let us remark that if $F_{1}^{\prime} \leq F_{1}$ and $F_{2}^{\prime} \geq F_{2}$, then $D_{\Phi}\left(F_{1}, F_{2}\right) \subseteq D_{\Phi}\left(F_{1}^{\prime}, F_{2}^{\prime}\right)$. Define $p: D_{\Phi} \rightarrow D(\hat{R}), p(x, y)=x$ the projection on the base of the special flow. We shall show that the limiting distribution $\mathrm{P}_{N_{1}, N_{2}}^{\prime}$ exists and it is given by

$$
\mathrm{P}_{N_{1}, N_{2}}^{\prime}\left((a, b) \times\left\{c_{-N_{1}+1}\right\} \times \cdots\left\{c_{0}\right\} \times \cdots \times\left\{c_{N_{2}}\right\}\right)=\tilde{\mu}\left(D_{\Phi}(\log a, \log b) \cap p^{-1} \mathcal{C}_{N_{1}, N_{2}}\right) .
$$

Consider $\varepsilon>0$. For each $n \in \mathbb{N}$, the collection $\left\{\mathcal{C}: \mathcal{C} \in \mathfrak{C}_{n, n}\right\}$ is a countable partition of $D(\hat{R})$. Let us choose $n \geq 4$ so that, by Lemma 3.6. we have $\left|g\left(\hat{\omega}^{\prime}\right)-g\left(\hat{\omega}^{\prime \prime}\right)\right| \leq \frac{\varepsilon}{2}$ for all $\hat{\omega}^{\prime}, \hat{\omega}^{\prime \prime} \in \mathcal{C}$. Define also $A_{\mathcal{C}}:=\left\{\hat{\omega} \in \mathcal{C}: a<\frac{\hat{q}_{\hat{n}_{L}(\hat{\omega})}(\hat{\omega})}{L}<b, \hat{R}^{\hat{n}_{L}(\hat{\omega})-1}(\hat{\omega}) \in \mathcal{C}_{N_{1}, N_{2}}\right\}$. By the second part of Lemma 3.7. there exists $L_{1}>0$ such that $\hat{n}_{L}(\alpha)>N$ for every $L \geq L_{1}$ and every $\alpha$ in the complement of a set of $\mu$-measure less than $\varepsilon$. Hence, (33) gives us

$$
\left|\mu\left(\left\{\alpha: a<\frac{\hat{q}_{\hat{n}_{L}(\alpha)}(\alpha)}{L}<b, \sigma_{\hat{n}_{L}(\alpha)+j}=c_{j},-N_{1}<j \leq N_{2}\right\}\right)-\sum_{\mathcal{C} \in \mathfrak{C}_{n, n}} \hat{\mu}\left(A_{\mathcal{C}}\right)\right| \leq 2 \varepsilon .
$$

Let us consider the finite collection of cylinders $\mathfrak{C}_{n, n}^{h, m}$ whose elements $\mathcal{C}=\mathcal{C}\left[c_{-n}, \ldots ; \ldots, c_{n}\right]$ are such that $c_{i}=h_{i} \cdot m_{i}^{ \pm} \in \Sigma$ with $h_{i}<h$ and $m_{i}<m$ for $-n \leq i \leq n$. It is clear that if $\mathcal{C} \in \mathfrak{C}_{n, n} \backslash \mathfrak{C}_{n, n}^{h, m}$, then there exist $-n \leq i \leq n$ such that $h_{i}>h$ or $m_{i}>m$ and therefore, by the $\hat{R}$-invariance of the measure $\hat{\mu}$ and Remark [1.7, we get

$$
\begin{aligned}
& \sum_{\mathcal{C} \in \mathfrak{C}_{n, n} \backslash \mathfrak{C}_{n, n}^{h, m}} \hat{\mu}(\mathcal{C}) \leq \sum_{i=-n}^{n} \sum_{\substack{l, j^{ \pm} \in \Sigma \\
l \geq h \text { or } j \geq m}} \hat{\mu}\left(\hat{R}^{i}\left(\hat{\mathcal{C}}\left[l \cdot j^{ \pm}\right]\right)\right) \leq \\
& \leq \frac{6(2 n+1)}{\log 3}\left(\sum_{l \geq h} \sum_{j \geq 1}+\sum_{l \geq 0} \sum_{j \geq m}\right) \frac{1}{4 l^{2}+8 l+3} \frac{1}{j^{2}} \leq \\
& \leq C_{13} \sum_{l \geq h} \frac{1}{4 l^{2}+8 l+3}+C_{14} \sum_{j \geq m} \frac{1}{j^{2}} \leq C_{15}\left(\frac{1}{h}+\frac{1}{m}\right),
\end{aligned}
$$

for some $C_{13}, C_{14}, C_{15}>0$. Thus, it is possible to choose $h$ and $m$ sufficiently large, so that $\sum_{\mathcal{C} \in \mathfrak{C}_{n, n} \backslash \mathfrak{C}_{n, n}^{h, m}} \hat{\mu}(\mathcal{C}) \leq \varepsilon$. For each $\mathcal{C} \in \mathfrak{C}_{n, n}^{h, m}$ we can find $L_{0}(\mathcal{C})$ and $U(\mathcal{C})$ as in Lemma 3.8 and for 
every $L \geq \max _{\mathcal{C} \in \mathfrak{C}_{n, n} \backslash \mathfrak{C}_{n, n}^{h, m}} L_{0}(\mathcal{C})$, using also the inclusion $A_{\mathcal{C}} \subseteq \mathcal{C}$ and (35), we get

$$
\begin{aligned}
\left|\mu\left(\left\{\alpha: a<\frac{\hat{q}_{\hat{n}_{L}(\alpha)}(\alpha)}{L}<b, \sigma_{\hat{n}_{L}(\alpha)+j}=c_{j},-N_{1}<j \leq N_{2}\right\}\right)-\sum_{\mathcal{C} \in \mathfrak{C}_{n, n}^{h, m}} \hat{\mu}\left(A_{\mathcal{C} \backslash U(\mathcal{C})}\right)\right| \leq \\
\quad \leq 2 \varepsilon+\left|\sum_{\mathcal{C} \in \mathfrak{C}_{n, n} \backslash \mathfrak{C}_{n, n}^{h, m}} \hat{\mu}\left(A_{\mathcal{C}}\right)+\sum_{\mathcal{C} \in \mathfrak{C}_{n, m}^{h, m}} \hat{\mu}\left(A_{\mathcal{C} \cap U(\mathcal{C})}\right)\right| \leq 3 \varepsilon+7 \varepsilon \sum_{\mathcal{C} \in \mathfrak{C}_{n, n}^{h, m}} \hat{\mu}(\mathcal{C}) \leq 10 \varepsilon .
\end{aligned}
$$

In order to get (34), it is enough to prove that, for each $\mathcal{C} \in \mathfrak{C}_{n, n}^{h, m}$ and sufficiently large $L$, we have

$$
\left|\frac{\hat{\mu}\left(A_{\mathcal{C} \backslash U(\mathcal{C})}\right)}{\hat{\mu}(\mathcal{C} \backslash U(\mathcal{C}))}-\tilde{\mu}\left(D_{\Phi}(\log a, \log b) \cap p^{-1} \mathcal{C}_{N_{1}, N_{2}}\right)\right| \leq C_{16} \varepsilon,
$$

for some $C_{16}>0$. Let $\mathcal{C} \in \mathfrak{C}_{n, n}^{h, m}$ be fixed, consider $T=\log L-g_{\mathcal{C}}$, and let $U=U(\mathcal{C})$ be as in Lemma 3.8. By the same Lemma, for $L \geq L_{0}(\mathcal{C})$, we have $\hat{n}_{L}(\hat{\omega})=r(\hat{\omega}, T)$ for every $\hat{\omega} \in \mathcal{C} \backslash U$. Using Lemma 3.5 we get

$$
\begin{aligned}
& \left\{\hat{\omega} \in \mathcal{C} \backslash U: a<\frac{\hat{q}_{\hat{n}_{L}(\hat{\omega})}(\hat{\omega})}{L}<b\right\}=\left\{\hat{\omega} \in \mathcal{C} \backslash U: \log a<\log \hat{q}_{r(\hat{\omega}, T)}(\hat{\omega})-\log L<\log b\right\}= \\
& =\left\{\hat{\omega} \in \mathcal{C} \backslash U: \log a<S_{r(\hat{\omega}, T)}(\psi)(\hat{\omega})-T+\varepsilon_{L, \mathcal{C}}(\hat{\omega})<\log b\right\}
\end{aligned}
$$

where $\varepsilon_{L, \mathcal{C}}(\hat{\omega}):=\varepsilon_{\hat{n}_{L}(\hat{\omega})}(\hat{\omega})-g_{\mathcal{C}}+g(\hat{\omega})$ and $\varepsilon_{\hat{n}_{L}(\hat{\omega})}(\hat{\omega})$ is defined as in (15, 16). It is possible to show, using Lemma 3.3 and (31), that $\left|\varepsilon_{L, \mathcal{C}}(\hat{\omega})\right| \leq 2 \varepsilon$ uniformly on $\mathcal{C} \backslash U$ (see [18] and the proof of Theorem 1.1 therein). Denoting by $v\left(\Phi_{t}(x, y)\right)$ the vertical component $y^{\prime}$ of $\Phi_{t}(x, y)=\left(x^{\prime}, y^{\prime}\right)$, by (4) and the equality $\hat{n}_{L}(\hat{\omega})=r(\hat{\omega}, T)$, we get $S_{r(\hat{\omega}, T)}(\psi)(\hat{\omega})-T=\psi\left(\hat{R}^{\hat{n}_{L}(\hat{\omega})-1}(\hat{\omega})\right)-v\left(\Phi_{T}(\hat{\omega}, 0)\right)$, which represent the vertical distance from $\Phi_{T}(\hat{\omega}, 0)$ and the roof function. Observing that $\hat{R}^{\hat{n}_{L}(\hat{\omega})-1}(\hat{\omega})=$ $p\left(\Phi_{T}(\hat{\omega}, 0)\right)$, the condition (33) can be rewritten as $p\left(\Phi_{T}(\hat{\omega}, 0)\right) \in \mathcal{C}_{N_{1}, N_{2}}$ and, recalling the inclusion properties of the sets $D_{\Phi}\left(F_{1}, F_{2}\right)$, we get

$$
\begin{aligned}
& A_{\mathcal{C} \backslash U} \subseteq(\mathcal{C} \backslash U \times\{0\}) \cap \Phi_{-T}\left(D_{\Phi}(\log a-2 \varepsilon, \log b+2 \varepsilon) \cap p^{-1} \mathcal{C}_{N_{1}, N_{2}}\right) \quad \text { and } \\
& A_{\mathcal{C} \backslash U} \supseteq(\mathcal{C} \backslash U \times\{0\}) \cap \Phi_{-T}\left(D_{\Phi}(\log a+2 \varepsilon, \log b-2 \varepsilon) \cap p^{-1} \mathcal{C}_{N_{1}, N_{2}}\right) .
\end{aligned}
$$

Now we use the same strategy used in the proof of Lemma 3.8, "thickening" the sets and applying the mixing of the special flow $\left\{\Phi_{t}\right\}_{t \in \mathbb{R}}$ (see again [18] for details). By Lemma 3.3 we can choose $0<\delta<\min \left\{\min _{\hat{\omega} \in \mathcal{C}} \psi(\hat{\omega}), \varepsilon\right\}$ and for each $\hat{\omega} \in A_{\mathcal{C} \backslash U}$ and $0 \leq z<\delta$ it is possible to show that $(\hat{\omega}, z) \in \Phi_{-T}\left(D_{\Phi}(\log a-2 \varepsilon-\delta, \log b+2 \varepsilon) \cap p^{-1} \mathcal{C}_{N_{1}, N_{2}} \cup D_{\Phi}^{\delta}\right)$, where $D_{\Phi}^{\delta}=D(\hat{R}) \times[0, \delta)$. We get

$$
\begin{aligned}
\delta \cdot \hat{\mu}\left(A_{\mathcal{C} \backslash U}\right) \leq \tilde{\mu}((\mathcal{C} \backslash U & \left.\times[0, \delta)) \cap \Phi_{-T}\left(D_{\Phi}(\log a-3 \varepsilon, \log b+2 \varepsilon) \cap p^{-1} \mathcal{C}_{N_{1}, N_{2}} \cup D_{\Phi}^{\delta}\right)\right) \leq \\
& \leq \delta \cdot \hat{\mu}(\mathcal{C} \backslash U) \cdot\left(\tilde{\mu}\left(D_{\Phi}(\log a-3 \varepsilon, \log b+2 \varepsilon) \cap p^{-1} \mathcal{C}_{N_{1}, N_{2}}\right)+2 \varepsilon\right),
\end{aligned}
$$

where (37) follows from the mixing property of the flow (Proposition 3.4) after observing that $\tilde{\mu}\left(D_{\Phi}^{\delta}\right) \leq \varepsilon$ and possibly enlarging $L_{0}$ so that if $L \geq L_{0}$, also $T=T(L, \mathcal{C})$ is sufficiently large. 
Again, reasoning as in the proof of Lemma 3.8, it is possible to show that for each $\hat{\omega} \in \mathcal{C} \backslash U$ and $0 \leq z<\delta$ such that $(\hat{\omega}, z) \in \Phi_{-T}\left(D_{\Phi}(\log a+2 \varepsilon, \log b-2 \varepsilon-\delta) \cap p^{-1} \mathcal{C}_{N_{1}, N_{2}} \backslash D_{\Phi}^{\delta}\right)$, we have $\hat{\omega} \in A_{\mathcal{C} \backslash U}$. This implies that

$$
\mathcal{C} \backslash U \times[0, \delta) \cap \Phi_{-T}\left(D_{\Phi}(\log a+2 \varepsilon, \log b-3 \varepsilon) \cap p^{-1} \mathcal{C}_{N_{1}, N_{2}} \backslash D_{\Phi}^{\delta}\right) \subseteq A_{\mathcal{C} \backslash U} \times[0, \delta) .
$$

Remark that for any measurable $D \subseteq D_{\Phi}$, we have $\tilde{\mu}\left(D \backslash D_{\Phi}^{\delta}\right) \geq \tilde{\mu}(D)-\varepsilon$. Now, using mixing and enlarging $L_{0}$ if needed, for $L \geq L_{0}$ we get

$$
\delta \cdot \hat{\mu}\left(A_{\mathcal{C} \backslash U}\right) \geq \delta \cdot \hat{\mu}(\mathcal{C} \backslash U) \cdot\left(\tilde{\mu}\left(D_{\Phi}(\log a+2 \varepsilon, \log b-3 \varepsilon) \cap p^{-1} \mathcal{C}_{N_{1}, N_{2}}\right)-2 \varepsilon\right) .
$$

Moreover, by Fubini Theorem, for $\left(C_{17}, C_{18}\right) \in\{(-3,2),(2,-3)\}$,

$$
\left|\tilde{\mu}\left(D_{\Phi}\left(\log a+C_{17} \varepsilon, \log b+C_{18} \varepsilon\right) \cap p^{-1} \mathcal{C}_{N_{1}, N_{2}}\right)-\tilde{\mu}\left(D_{\Phi}(\log a, \log b) \cap p^{-1} \mathcal{C}_{N_{1}, N_{2}}\right)\right| \leq C_{19} \varepsilon,
$$

for some $C_{19}>0$. Finally, by (37] 39 ) we get (36) concluding thus the proof of the existence of the limiting distribution.

Remark 4.1. The set $\mathcal{C}_{N_{1}, N_{2}}$ in the previous proof can be replaced by any set of positive $\hat{\mu}$ measure in the base $D(\hat{R})$.

Now we give the proof of our Main Theorem as a corollary of Theorem 1.6.

Proof of the Main Theorem. Given $a, b \geq 1, a<b, N_{1}, N_{2} \in \mathbb{N}$ and $d_{j} \in \Omega,-N_{1}<j \leq N_{2}$, we want to write

$$
\mu\left(\left\{\alpha: a<\frac{q_{n_{L}}}{L}<b, \omega_{n_{L}+j}=d_{j},-N_{1}<j \leq N_{2}\right\}\right)
$$

in terms of analogous quantities for $\frac{\hat{q}_{\hat{n}_{L}}}{L}$.

Denoting by $\nu=\nu_{\hat{n}_{L}-1}$, we get $\nu_{\hat{n}_{L}}=\nu+\tau+1$, where $\tau=\tau\left(R^{\hat{n}_{L}-1}\left(\hat{\omega}^{+}\right)\right)$. By construction we have

$$
\hat{q}_{\hat{n}_{L}-1}=q_{\nu}<q_{n_{L}} \leq q_{\nu+\tau+1}=\hat{q}_{\hat{n}_{L}}
$$

and therefore $q_{n_{L}}=q_{\nu+j}$ for some $1 \leq j \leq \tau+1$. Notice that, by definition of $\hat{n}_{L}$, we have $0<\frac{\hat{q}_{\hat{n}_{L}-1}}{L}<1$.

We distinguish three cases: (i) $j=\tau+1$, (ii) $j=\tau$ and (iii) $1 \leq j \leq \tau-1$. Let us remark that in the first case we have $q_{n_{L}}=\hat{q}_{\hat{n}_{L}}$. For cases (ii) and (iii), by (뜨), we observe that

$$
\left[\begin{array}{cccccc}
2 k_{\nu+\tau+1} & \xi_{\nu+\tau} & & & & \\
-1 & 2 k_{\nu+\tau} & -1 & & & \\
& -1 & 2 & \ddots & & \\
& & -1 & \ddots & -1 & \\
& & & \ddots & 2 & -1 \\
& & & & -1 & 2
\end{array}\right] \cdot\left[\begin{array}{c}
q_{\nu+\tau} \\
q_{\nu+\tau-1} \\
\vdots \\
q_{\nu+j} \\
\vdots \\
q_{\nu+2} \\
q_{\nu+1}
\end{array}\right]=\left[\begin{array}{c}
q_{\nu+\tau+1} \\
0 \\
\vdots \\
0 \\
\vdots \\
0 \\
q_{\nu}
\end{array}\right]
$$


and therefore $q_{\nu+j}=C^{(1)} q_{\nu+\tau+1}+C^{(2)} q_{\nu}$, with $C^{(i)}=C^{(i)}\left(j, k_{\nu+\tau}, \xi_{\nu+\tau}, k_{\nu+\tau+1}\right)$. It can be shown that $0<C^{(1)}, C^{(2)}<1$, except for $j=\tau$ and $\xi_{\nu+\tau}=+1$ when we have $-1<C^{(2)}<0$ (see case (ii') below).

We can assume $N_{1} \geq 2$. Indeed the case $N_{1}=1$ can be recovered by $N_{1}=2$ considering the sum over all possible values of $\omega_{n_{L}-1}$. The values of $d_{-1}, d_{0} \in \Omega$ determine the case (i), (ii) or (iii) we are dealing with:

\begin{tabular}{|c|c|c|}
\hline$d_{-1}$ & $d_{0}$ & case \\
\hline \hline$\neq 1^{-}$ & $\in \Omega$ & $(\mathrm{i})$ \\
\hline$=1^{-}$ & $\begin{array}{c}=m^{+} \\
=m^{-}(m \neq 1)\end{array}$ & $\begin{array}{c}\left(\text { ii' }^{\prime}\right) \\
(\text { ii' })\end{array}$ \\
\hline$=1^{-}$ & $=1^{-}$ & (iii) \\
\hline
\end{tabular}

For each case we can rewrite (40) as follows:

(i) There exist $N_{1}^{\prime}, N_{2}^{\prime} \in \mathbb{N}$ such that

$$
\begin{aligned}
& \mu\left(\left\{\alpha: a<\frac{q_{n_{L}}}{L}<b, \omega_{n_{L}+j}=d_{j},-N_{1}<j \leq N_{2}\right\}\right)= \\
& =\sum_{c} \mu\left(\left\{\alpha: a<\frac{\hat{q}_{\hat{n}_{L}}}{L}<b, \sigma_{\hat{n}_{L}+j}=c_{j},-N_{1}^{\prime}<j \leq N_{2}^{\prime}\right\}\right),
\end{aligned}
$$

where the sum is taken over those $c=\left\{c_{j}\right\} \in \Sigma^{N_{1}^{\prime}+N_{2}^{\prime}}$ that, after being coded into the alphabet $\Omega$, are compatible with the $\left\{d_{j}\right\} \in \Omega^{N_{1}+N_{2}}$. Notice that, if $c_{j}=h_{j} \cdot m_{j}^{ \pm} \in \Sigma,-N_{1}^{\prime}<j \leq N_{2}^{\prime}$, the coding of $c=\left\{c_{j}\right\}$ into the alphabet $\Omega$ gives us a sequence of length $\sum_{j=-N_{1}^{\prime}+1}^{N_{2}^{\prime}}\left(h_{j}+1\right)$.

(ii') There exist $N_{1}^{\prime}, N_{2}^{\prime} \in \mathbb{N}$ such that

$$
\begin{aligned}
& \mu\left(\left\{\alpha: a<\frac{q_{n_{L}}}{L}<b, \omega_{n_{L}+j}=d_{j},-N_{1}<j \leq N_{2}\right\}\right)= \\
& =\sum_{c} \mu\left(\left\{\alpha: \frac{a+C^{(2)}}{C^{(1)}}<\frac{\hat{q}_{\hat{n}_{L}}}{L}<\frac{b}{C^{(1)}}, \sigma_{\hat{n}_{L}+j}=c_{j},-N_{1}^{\prime}<j \leq N_{2}^{\prime}\right\}\right),
\end{aligned}
$$

where the sum is taken over those $c=\left\{c_{j}\right\} \in \Sigma^{N_{1}^{\prime}+N_{2}^{\prime}}$ as in (41). Notice that $C^{(1)}$ and $C^{(2)}$ depend on $c$ via $k_{\nu+\tau+1}, k_{\nu+\tau}$ and $\xi_{\nu+\tau}$.

(ii") \& (iii) There exist $N_{1}^{\prime}, N_{2}^{\prime} \in \mathbb{N}$ such that

$$
\begin{aligned}
& \mu\left(\left\{\alpha: a<\frac{q_{n_{L}}}{L}<b, \omega_{n_{L}+j}=d_{j},-N_{1}<j \leq N_{2}\right\}\right)= \\
& =\sum_{c} \mu\left(\left\{\alpha: \frac{a}{C^{(1)}}<\frac{\hat{q}_{\hat{n}_{L}}}{L}<\frac{b-C^{(2)}}{C^{(1)}}, \sigma_{\hat{n}_{L}+j}=c_{j},-N_{1}^{\prime}<j \leq N_{2}^{\prime}\right\}\right),
\end{aligned}
$$

where the sum is taken over those $c=\left\{c_{j}\right\} \in \Sigma^{N_{1}^{\prime}+N_{2}^{\prime}}$ as in (41), with the further constraint that $C^{(2)}<b-a$. 
Now, we want to consider the limit as $L \rightarrow \infty$ in (41), (42) and (43). Let us show that in each case we have uniform convergence of the series. Denoting

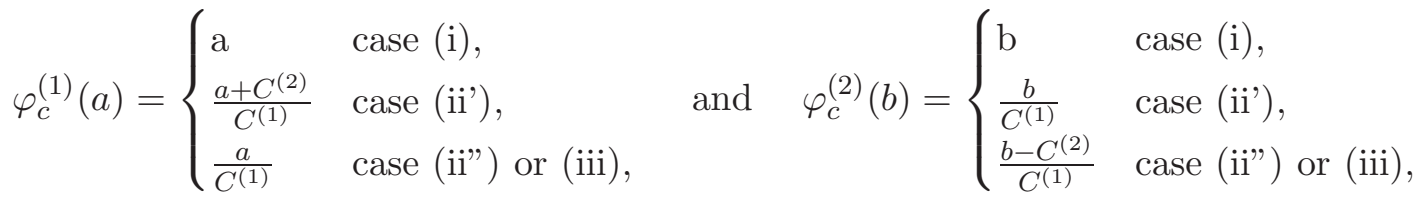

we get

$$
\begin{aligned}
\mu\left(\left\{\alpha: \varphi_{c}^{(1)}(a)\right.\right. & \left.\left.<\frac{\hat{q}_{\hat{n}_{L}}}{L}<\varphi_{c}^{(2)}(b), \sigma_{\hat{n}_{L}+j}=c_{j},-N_{1}^{\prime}<j \leq N_{2}^{\prime}\right\}\right) \leq \\
& \leq \mu\left(\left\{\alpha: R^{\hat{n}_{L}(\alpha)-1}(\alpha) \in \mathcal{C}_{N_{1}^{\prime}, N_{2}^{\prime}}^{\prime}\right\}\right)=\mu\left(\mathcal{C}_{N_{1}^{\prime}, N_{2}^{\prime}}^{\prime}\right)
\end{aligned}
$$

where $\mathcal{C}_{N_{1}^{\prime}, N_{2}^{\prime}}^{\prime}:=R^{N_{1}^{\prime}-1}\left(\mathcal{C}\left[c_{-N_{1}^{\prime}+1}, \ldots, c_{0}, \ldots, c_{N_{2}^{\prime}}\right]\right)$. Now, if $c_{j}=h_{j} \cdot m_{j}^{ \pm}$, by Remark 1.7] we obtain the estimate

$$
\mu\left(\mathcal{C}_{N_{1}^{\prime}, N_{2}^{\prime}}^{\prime}\right)=\mu\left(\mathcal{C}\left[c_{-N_{1}^{\prime}+1}, \ldots, c_{0}, \ldots, c_{N_{2}^{\prime}}\right]\right) \leq C_{20} \prod_{j=-N_{1}^{\prime}+1}^{N_{2}^{\prime}} \frac{1}{\left(4 h_{j}^{2}+8 h_{j}+3\right) m_{j}^{2}},
$$

for some constant $C_{20}>0$. Now the series of suprema is controlled as follows:

$$
\begin{aligned}
& \quad \sum_{\substack{c_{i}=h_{j} \cdot m_{i}^{ \pm} \in \Sigma \\
j=-N_{1}^{\prime}+1, \ldots, N_{2}^{\prime}}} \sup _{L}\left[\mu\left(\left\{\alpha: \varphi_{c}^{(1)}(a)<\frac{\hat{q}_{\hat{n}_{L}}}{L}<\varphi_{c}^{(2)}(b), \sigma_{\hat{n}_{L}+j}=c_{j},-N_{1}^{\prime}<j \leq N_{2}^{\prime}\right\}\right)\right] \leq \\
& \leq 2 C_{20} \sum_{h_{-N_{1}^{\prime}+1}, \ldots, h_{N_{2}^{\prime}} \geq 0} \sum_{m_{-N_{1}^{\prime}+1}, \ldots, m_{N_{2}^{\prime}} \geq 1} \prod_{j=-N_{1}^{\prime}+1}^{N_{2}^{\prime}} \frac{1}{\left(4 h_{j}^{2}+8 h_{j}+3\right) m_{j}^{2}} \leq C_{21},
\end{aligned}
$$

for some constant $C_{21}>0$. Now, because of uniform convergence, we can interchange the limit as $L \rightarrow \infty$ and the series in (41), (42) and (43) and by Theorem 1.6 we get

$$
\begin{aligned}
& \lim _{L \rightarrow \infty} \mu\left(\left\{\alpha: a<\frac{q_{n_{L}}}{L}<b, \omega_{n_{L}+j}=d_{j},-N_{1}<j \leq N_{2}\right\}\right)= \\
& =\sum_{c} \mathrm{P}_{N_{1}^{\prime}, N_{2}^{\prime}}^{\prime}\left(\left(\varphi_{c}^{(1)}(a), \varphi_{c}^{(2)}(b)\right) \times\left\{c_{-N_{1}^{\prime}+1}\right\} \times \cdots \times\left\{c_{0}\right\} \times \cdots \times\left\{c_{N_{2}^{\prime}}\right\}\right)<\infty
\end{aligned}
$$

concluding thus the proof of our Main Theorem.

\section{Appendix A}

Before giving the proof of Proposition 3.4, we present, an useful Lemma and its Corollary.

Lemma 4.2. If $\left\{p_{i} / q_{i}\right\}$ are the ECF-convergents of $\alpha=\left[\left[\left(k_{1}, \xi_{1}\right),\left(k_{2}, \xi_{2}\right), \ldots\right]\right]$, then for any $m \geq 2$

$$
\begin{aligned}
& {\left[\left[\left(k_{m}, \xi_{m-1}\right),\left(k_{m-1}, \xi_{m-2}\right), \ldots,\left(k_{2}, \xi_{1}\right),\left(k_{1}, *\right)\right]\right]=\frac{q_{m-1}}{q_{m}},} \\
& {\left[\left[\left(k_{m}, \xi_{m-1}\right),\left(k_{m-1}, \xi_{m-2}\right), \ldots,\left(k_{3}, \xi_{2}\right),\left(k_{2}, *\right)\right]\right]=\frac{p_{m-1}}{p_{m}} .}
\end{aligned}
$$


Corollary 4.3. Given $\left\{\left(k_{n}, \xi_{n}\right)\right\}_{n \in \mathbb{Z}}$, let us consider $m \geq 2, \beta \in(0,1] \backslash \mathbb{Q}$ and $\gamma \in[-1,1] \backslash \mathbb{Q}$ such that

$$
\begin{aligned}
& \beta=\left[\left[\left(k_{m}, \xi_{m-1}\right),\left(k_{m-1}, \xi_{m-2}\right), \ldots,\left(k_{2}, \xi_{1}\right),\left(k_{1}, \xi_{0}\right),\left(k_{0}, \xi_{-1}\right), \ldots\right]\right], \\
& \gamma=\left[\left[\left(0, \xi_{0}\right) ;\left(k_{0}, \xi_{-1}\right),\left(k_{-1}, \xi_{-2}\right),\left(k_{-2}, \xi_{-3}\right), \ldots\right]\right]
\end{aligned}
$$

and let $\left\{p_{i} / q_{i}\right\}$ be the ECF-convergents of $\left[\left[\left(k_{1}, \xi_{1}\right),\left(k_{2}, \xi_{2}\right),\left(k_{3}, \xi_{3}\right) \ldots\right]\right]$. Then

$$
\beta=\frac{q_{m-1}+p_{m-1} \cdot \gamma}{q_{m}+p_{m} \cdot \gamma}
$$

We skip the proofs of Lemma 4.2 and Corollary 4.3 since they can be recovered, mutatis mutandis, from proofs of the analogous results for Euclidean expansions. Now we are ready to give the following

\section{Proof of Proposition 3.4.}

This proof imitates the one given in [7] (and reviewed in [18]) concerning Euclidean expansions. In order to prove the statement, we shall perform two steps: 1) construct the global stable and unstable foliations, 2) prove that they form a non-integrable pair (see e.g. [2], Chapter I or [7], $\S 2.4$ ). Their non integrability will imply that the Pinsker partition [17] is trivial and hence $\left\{\Phi_{t}\right\}_{t}$ is a $\mathrm{K}$-flow and, in particular, it is mixing.

Given $\left(\hat{\omega}^{(0)}, y_{0}\right) \in D_{\Phi}$, let us construct the local stable and unstable leaves through it, denoted by $\Gamma_{\text {loc }}^{(s)}\left(\hat{\omega}^{(0)}, y_{0}\right)$ and $\Gamma_{\text {loc }}^{(u)}\left(\hat{\omega}^{(0)}, y_{0}\right)$ respectively. As a reference, see e.g. [17].

Since the roof function $\psi(\hat{\omega})$ depends only on $\left(\ldots, \sigma_{-1}, \sigma_{0} ; \sigma_{1}, \sigma_{2}\right), \sigma_{i} \in \Sigma$, it is clear that the local unstable leaf is given by a piece of segment in the $\hat{\omega}^{+}$-direction:

$$
\Gamma_{\text {loc }}^{(u)}\left(\hat{\omega}^{(0)}, y_{0}\right) \subset\left\{\left(\hat{\omega}, y_{0}\right):\left(\hat{R}^{2} \hat{\omega}\right)^{-}=\left(\hat{R}^{2} \hat{\omega}^{(0)}\right)^{-}\right\} \subset\left\{\left(\hat{\omega}, y_{0}\right): \hat{\omega}^{-}=\left(\hat{\omega}^{(0)}\right)^{-}\right\} .
$$

The local stable leaf $\Gamma_{\text {loc }}^{(s)}\left(\hat{\omega}^{(0)}, y_{0}\right)$ is given by those pairs $(\hat{\omega}, y)$ satisfying

$$
\left\{\begin{array}{l}
\hat{\omega}^{+}=\left(\hat{\omega}^{(0)}\right)^{+} \\
y=y_{0}+\log \left(\frac{2 k_{1}+\left(\hat{\omega}^{(0)}\right)^{-}}{2 k_{1}+\hat{\omega}^{-}}\right)+\log \left(\frac{1+\left(\hat{\omega}^{(0)}\right)^{+} \cdot \hat{\omega}^{-}}{1+\left(\hat{\omega}^{(0)}\right)^{+} \cdot\left(\hat{\omega}^{(0)}\right)^{-}}\right) .
\end{array}\right.
$$

In order to see this, let us denote $\left(\hat{\omega}^{(t)}, y_{t}\right)=\Phi_{t}\left(\hat{\omega}^{(0)}, y_{0}\right)$ and consider a small segment in the $\hat{\omega}^{-}$-direction through it:

$$
\Gamma_{\delta_{t}}^{t}=\left\{\left(\hat{\omega}, y_{t}\right): \hat{\omega}^{+}=\left(\hat{\omega}^{(t)}\right)^{+},\left|\hat{\omega}^{-}-\left(\hat{\omega}^{(t)}\right)^{-}\right|<\delta_{t}\right\}
$$

where $\delta_{t}$ is chosen sufficiently small so that

$$
\Phi_{-t}\left(\Gamma_{\delta_{t}}^{t}\right) \subset\left\{(\hat{\omega}, y): \hat{\omega}^{+}=\left(\hat{\omega}^{(0)}\right)^{+},\left|y-y_{0}\right|<\delta, 0<y_{0}-\delta<y<\varphi(\hat{\eta})-\delta\right\}
$$

for some $\delta>0$.

Now, if $(\hat{\omega}, y) \in \Phi_{-t}\left(\Gamma_{\delta_{t}}^{t}\right)$, then by construction $r(\hat{\omega}, t)=r\left(\hat{\omega}^{(0)}, t\right)=: r(t)$ and, from the definition 
(44) of the special flow, $y-S_{r(t)-1}(\psi, \hat{R})(\hat{\omega})=y_{t}-t=y_{0}-S_{r(t)-1}(\psi, \hat{R})\left(\hat{\omega}^{(0)}\right)$. From (13) and (44), because of telescopic cancellations, we get

$$
S_{r(t)-1}(\psi, \hat{R})(\hat{\omega})=\sum_{l=2}^{\nu} \log \left[\left[\left(k_{l}, \xi_{l-1}\right),\left(k_{l-1}, \xi_{l-2}\right), \ldots\right]\right]^{-1}=\frac{q_{\nu}+p_{\nu} \cdot \hat{\omega}^{-}}{q_{1}+p_{1} \cdot \hat{\omega}^{-}}
$$

where $\nu=\nu_{r(t)-1}$ and $\left\{p_{i} / q_{i}\right\}$ are the ECF-convergents of $\left(\hat{\omega}^{(0)}\right)^{+}$. Similarly we find

$$
S_{r(t)-1}(\psi, \hat{R})\left(\hat{\omega}^{(0)}\right)=\frac{q_{\nu}+p_{\nu} \cdot\left(\hat{\omega}^{(0)}\right)^{-}}{q_{1}+p_{1} \cdot\left(\hat{\omega}^{(0)}\right)^{-}}
$$

and therefore, recalling that $p_{1}=1$ and $q_{1}=2 k_{1}$,

$$
\begin{aligned}
y & =y_{0}+S_{r(t)-1}(\psi, \hat{R})(\hat{\omega})-S_{r(t)-1}(\psi, \hat{R})\left(\hat{\omega}^{(0)}\right)= \\
& =y_{0}+\log \left(\frac{2 k_{1}+\left(\hat{\omega}^{(0)}\right)^{-}}{2 k_{1}+\hat{\omega}^{-}}\right)+\log \left(\frac{1+\frac{p_{\nu}}{q_{\nu}} \hat{\omega}^{-}}{1+\frac{p_{\nu}}{q_{\nu}}\left(\hat{\omega}^{(0)}\right)^{-}}\right) .
\end{aligned}
$$

As $t \rightarrow \infty$, also $\nu \rightarrow \infty$ and $\frac{p_{\nu}}{q_{\nu}} \rightarrow\left(\hat{\omega}^{(0)}\right)^{+}$; therefore (47) leads us to (46).

The global unstable and stable leaves are obtained as

$$
\Gamma^{(u)}\left(\hat{\omega}^{(0)}, y_{0}\right)=\bigcup_{t} \Phi_{t} \Gamma_{\mathrm{loc}}^{(u)}\left(\hat{\omega}^{(-t)}, y_{-t}\right), \quad \Gamma^{(s)}\left(\hat{\omega}^{(0)}, y_{0}\right)=\bigcup_{t} \Phi_{-t} \Gamma_{\mathrm{loc}}^{(u)}\left(\hat{\omega}^{(t)}, y_{t}\right) .
$$

Let us consider a sufficiently small neighborhood $\mathcal{U}^{(0)} \subset D_{\Phi}$ of $\left(\hat{\omega}^{(0)}, y_{0}\right)$. In order to prove the non integrability of the stable and unstable foliations, it is enough to show that, for a set of positive measure of $(\hat{\omega}, y) \in \mathcal{U}^{(0)},(\hat{\omega}, y)$ can be connected to $\left(\hat{\omega}^{(0)}, y_{0}\right)$ through a polygonal made of segments of stable and unstable leaves. This is achieved in particular if there exist $\left(\hat{\omega}^{\prime}, y^{\prime}\right),\left(\hat{\omega}^{\prime \prime}, y^{\prime \prime}\right) \in \mathcal{U}^{(0)}$, such that $\left(\hat{\omega}^{\prime}, y^{\prime}\right) \in \Gamma^{(s)}\left(\hat{\omega}^{(0)}, y_{0}\right),\left(\hat{\omega}^{\prime \prime}, y^{\prime \prime}\right) \in \Gamma^{(u)}\left(\hat{\omega}^{\prime}, y^{\prime}\right)$ and $(\hat{\omega}, y) \in \Gamma^{(s)}\left(\hat{\omega}^{\prime \prime}, y^{\prime \prime}\right)$. Using the explicit local parametrizations (45,46), one can check that these points exist as soon as one can find $\left(\hat{\omega}^{\prime}\right)^{-}$ and $y^{\prime}$ such that

$$
\left\{\begin{array}{l}
y^{\prime}=y_{0}+\log \left(\frac{2 k_{1}+\left(\hat{\omega}^{(0)}\right)^{-}}{2 k_{1}+\left(\hat{\omega}^{\prime}\right)^{-}}\right)+\log \left(\frac{1+\left(\hat{\omega}^{(0)}\right)^{+} \cdot\left(\hat{\omega}^{\prime}\right)^{-}}{1+\left(\hat{\omega}^{(0)}\right)^{+} \cdot\left(\hat{\omega}^{(0)}\right)^{-}}\right), \\
y=y^{\prime}+\log \left(\frac{2 k_{1}+\left(\hat{\omega}^{\prime}\right)^{-}}{2 k_{1}+\hat{\omega}^{-}}\right)+\log \left(\frac{1+\hat{\omega}^{+} \cdot \hat{\omega}^{-}}{1+\hat{\omega}^{+} \cdot\left(\hat{\omega}^{\prime}\right)^{-}}\right) .
\end{array}\right.
$$

A direct computation shows that equations (48) in the unknowns $\left(\hat{\omega}^{\prime}\right)^{-}$and $y^{\prime}$ can be solved when

$$
\frac{\hat{\omega}^{+}\left(2 k_{1}+\hat{\omega}^{-}\right) e^{y}}{1+\hat{\omega}^{-} \cdot \hat{\omega}^{+}} \neq \frac{\left(\hat{\omega}^{(0)}\right)^{+}\left(2 k_{1}+\left(\hat{\omega}^{(0)}\right)^{-}\right) e^{y_{0}}}{1+\left(\hat{\omega}^{(0)}\right)^{-} \cdot\left(\hat{\omega}^{(0)}\right)^{+}},
$$

and (49) holds true for $(\hat{\omega}, y)$ in a subset of $\mathcal{U}^{(0)}$ with positive measure, as desired.

\section{Acknowledgments}

I would like to thank my advisor Prof. Ya. G. Sinai for his constant guidance and invaluable encouragement. Thanks also to C. Ulcigrai for many fruitful conversations and to the anonymous referee for his/her thorough remarks. Lastly, I would like to dedicate this work to the memory of Riccardo Venier. 


\section{References}

[1] R. L. Adler. Continued fractions and Bernoulli trials. In: Ergodic theory. Moser, J., Phillips, E., Varadhan, S. (eds.). Lecture notes. New York: Courant Inst. Math. Sci. 1975.

[2] D. V. Anosov. Geodesic flows on closed Riemannian manifolds of negative curvature. Trudy Mat. Inst. Steklov., 90:209, 1967.

[3] M. V. Berry and J. Goldberg. Renormalisation of curlicues. Nonlinearity, 1(1):1-26, 1988.

[4] R. Bowen. Invariant measures for Markov maps of the interval. Comm. Math. Phys., 69(1):117, 1979. With an afterword by Roy L. Adler and additional comments by Caroline Series.

[5] I. P. Cornfeld, S. V. Fomin, and Ya. G. Sinai. Ergodic theory, volume 245 of Grundlehren der Mathematischen Wissenschaften [Fundamental Principles of Mathematical Sciences]. SpringerVerlag, New York, 1982.

[6] E. A. Coutsias and N. D. Kazarinoff. The approximate functional formula for the theta function and Diophantine Gauss sums. Trans. Amer. Math. Soc., 350(2):615-641, 1998.

[7] E. I. Dinaburg and Ya. G. Sinai. Statistics of the solutions of the integral equation $a x-b y= \pm 1$ (Russian). Funktsional. Anal. i Prilozhen., 24(3):1-8, 96, 1990. English Translation in Funct. Anal. Appl. 24 (1990), 3:165-171, 1991.

[8] A. Fedotov and F. Klopp. Renormalization of exponential sums and matrix cocycles. In Séminaire: Équations aux Dérivées Partielles. 2004-2005, pages Exp. No. XVI, 12. École Polytech., Palaiseau, 2005.

[9] M. J. Feigenbaum. Presentation functions, fixed points, and a theory of scaling function dynamics. J. Statist. Phys., 52(3-4):527-569, 1988.

[10] A. Ya. Khinchin. Continued fractions. The University of Chicago Press, Chicago, Ill.-London, 1964.

[11] C. Kraaikamp and A. O. Lopes. The theta group and the continued fraction expansion with even partial quotients. Geom. Dedicata, 59(3):293-333, 1996.

[12] V. A. Rokhlin. Exact endomorphisms of a Lebesgue space. Izv. Akad. Nauk SSSR Ser. Mat., 25:499-530, 1961. English translation in Amer. Math. Soc. Transl. (2) 39:1-36, 1964.

[13] H. G. Schuster. Deterministic chaos. VCH Verlagsgesellschaft mbH, Weinheim, augmented edition, 1995. An introduction.

[14] F. Schweiger. Continued fractions with odd and even partial quotients. Arbeitsberichte Math. Institut Universtät Salzburg, 4:59-70, 1982.

[15] F. Schweiger. On the approximation by continues fractions with odd and even partial quotients. Arbeitsberichte Math. Institut Universtät Salzburg, 1-2:105-114, 1984. 
[16] F. Schweiger. Ergodic theory of fibred systems and metric number theory. Oxford Science Publications. The Clarendon Press Oxford University Press, New York, 1995.

[17] Ya. G. Sinai. Topics in Ergodic Theory, volume 44 of Princeton Mathematical Series. Princeton University Press, Princeton, NJ, 1994.

[18] Ya. G. Sinai and C. Ulcigrai. Renewal-type limit theorem for the Gauss map and continued fractions. Ergodic Theory and Dynamical Systems, 28:643-655, 2008.

[19] B. Vallée. Euclidean dynamics. Discrete Contin. Dyn. Syst., 15(1):281-352, 2006. 